\title{
Electron spin diffusion and transport in graphene
}

\author{
P. Zhang and M. W. Wu* \\ Hefei National Laboratory for Physical Sciences at Microscale and Department of Physics, \\ University of Science and Technology of China, Hefei, Anhui, 230026, China
}

(Dated: October 29, 2018)

\begin{abstract}
We investigate the spin diffusion and transport in a graphene monolayer on $\mathrm{SiO}_{2}$ substrate by means of the microscopic kinetic spin Bloch equation approach. The substrate causes a strong Rashba spin-orbit coupling field $\sim 0.15 \mathrm{meV}$, which might be accounted for by the impurities initially present in the substrate or even the substrate-induced structure distortion. By surface chemical doping with $\mathrm{Au}$ atoms, this Rashba spin-orbit coupling is further strengthened as the adatoms can distort the graphene lattice from $s p^{2}$ to $s p^{3}$ bonding structure. By fitting the Au doping dependence of spin relaxation from Pi et al. [Phys. Rev. Lett. 104, 187201 (2010)], the Rashba spin-orbit coupling coefficient is found to increase approximately linearly from 0.15 to $0.23 \mathrm{meV}$ with the increase of $\mathrm{Au}$ density. With this strong spin-orbit coupling, the spin diffusion or transport length is comparable with the experimental values. In the strong scattering limit (dominated by the electron-impurity scattering in our study), the spin diffusion is uniquely determined by the Rashba spin-orbit coupling strength and insensitive to the temperature, electron density as well as scattering. With the presence of an electric field along the spin injection direction, the spin transport length can be modulated by either the electric field or the electron density. It is shown that the spin diffusion and transport show an anisotropy with respect to the polarization direction of injected spins. The spin diffusion or transport lengths with the injected spins polarized in the plane defined by the spin-injection direction and the direction perpendicular to the graphene are identical, but longer than that with the injected spins polarized vertical to this plane. This anisotropy differs from the one given by the two-component drift-diffusion model, which indicates equal spin diffusion or transport lengths when the injected spins are polarized in the graphene plane and relatively shorter lengths when the injected spins are polarized perpendicular to the graphene plane.
\end{abstract}

PACS numbers: 72.25.Rb, 75.40.Gb, 72.80.Vp, 71.70.Ej

\section{INTRODUCTION}

Graphene is considered to be a promising candidate for the spintronic applications recently, ${ }^{1-22}$ partly due to the perfect two dimensionality, gate-voltage-tunable charge carrier type and density, ${ }^{3,4}$ high mobility ${ }^{5-7,23,24}$ as well as the potentially long spin relaxation time limited by the small intrinsic spin-orbit and hyperfine couplings. ${ }^{8-12,25,26}$ From the high mobility and long spin relaxation time, a long spin relaxation length, favorable to the spin information transport and manipulation, is anticipated. However, both the spin relaxation time and transport length were experimentally found to be much smaller than expected..$^{2,25,27-32}$ This suggests that the spin relaxation in the experiments is most likely to be contributed by extrinsic factors such as the possible impurity-enhanced spin relaxation ${ }^{28,30}$ via the ElliotYafet $^{33}$ mechanism or the enhanced Rashba spin-orbit coupling field ${ }^{9,11}$ from the impurities. ${ }^{26,34,35}$ The former case may exist in a highly dirty graphene sample and causes the spin relaxation time $\tau_{s}$ to be proportional to the momentum relaxation time $\tau_{p} .{ }^{28,30}$ However, for the latter case, the Dyakonov-Perel (DP) spin relaxation mechanism $^{36}$ dominates and the relation $\tau_{s} \propto \tau_{p}$ is absent. In fact, recently $\mathrm{Pi}$ et al. reported that $\tau_{s}$ increases with decreasing $\tau_{p}$ in the surface chemical doping experiment with $\mathrm{Au}$ atoms on graphene, ${ }^{31}$ indicating that the DP spin relaxation mechanism is important there. How- ever, the relation $\tau_{s} \propto 1 / \tau_{p}$, valid when the $\mathrm{DP}$ spin relaxation mechanism is dominant and the scattering is strong enough, is not obeyed in their experiment. ${ }^{31} \mathrm{Nev}$ ertheless, we will show that this deviation can be understood by taking account of the strengthening of the Rashba spin-orbit coupling with the increasing coverage of Au adatoms. The Rashba spin-orbit coupling, referred to as an extrinsic one, is due to the broken of the inversion symmetry which can be caused by either a perpendicular electric field, the interaction with substrate, or the atoms adsorbed on the surface..$^{9,11,26,34,35}$ The contribution of the electric field to the Rashba spin-orbit coupling is small $(\sim \mu \mathrm{eV}$ under a perpendicular electric field as large as $1 \mathrm{~V} / \mathrm{nm}),{ }^{26,32}$ while the adatoms can effectively enhance the Rashba spin-orbit coupling to be of order of $10 \mathrm{meV}$ by distorting the graphene lattice from $s p^{2}$ to $s p^{3}$ bonding structure. ${ }^{26,34,35}$

In this work, we investigate the spin diffusion and transport limited by the DP mechanism in a graphene monolayer on $\mathrm{SiO}_{2}$ substrate as presented by $\mathrm{Pi}$ et al.. ${ }^{31}$ To account for the short spin relaxation time $(\sim 70 \mathrm{ps})$ before $\mathrm{Au}$ doping in the experiment, ${ }^{31}$ we assume that the impurities inevitably present in the substrate, as well as the other effects such as the substrate-induced structure distortion, cause a strong Rashba spin-orbit coupling. When the surface chemical doping by Au atoms ${ }^{31}$ is performed, the Rashba spin-orbit coupling coefficient $\alpha_{\mathrm{R}}$ is further strengthened. By fitting the chemical dop- 
ing dependence of spin relaxation time from the experimental data, ${ }^{31}$ we obtain the chemical doping dependence of $\alpha_{\mathrm{R}}$. It is found that $\alpha_{\mathrm{R}}$ increases approximately linearly with the density of adatoms when the latter is not too high. With this essential information obtained, we then study the spin diffusion and transport in the graphene layer. The method utilized in our study is the kinetic spin Bloch equation (KSBE) approach which has been successfully applied to the study of spin dynamics in semiconductors. ${ }^{37}$ In the framework of this approach, the spatial spin precession frequency during the steadystate scattering-free spin diffusion (assumed to be along the $x$-axis) is ${ }^{37-41}$

$$
\boldsymbol{\omega}_{\mathbf{k}}=\left(2 \boldsymbol{\Omega}_{\mathbf{k}}+g \mu_{B} \mathbf{B}\right) / \partial_{k_{x}} \varepsilon_{\mathbf{k}} .
$$

Here $\boldsymbol{\Omega}_{\mathbf{k}}$ is the DP term, B is the external magnetic field and $\varepsilon_{\mathbf{k}}$ is the electron energy spectrum. The momentum dependence of $\boldsymbol{\omega}_{\mathbf{k}}$ leads to the inhomogeneous broadening in spin precession, with which any scattering (including the Coulomb scattering) can cause an irreversible spin relaxation along with spin diffusion and transport. ${ }^{37-41}$ It is noted that different DP terms as well as different energy spectra lead to distinct momentum dependences of $\boldsymbol{\omega}_{\mathbf{k}}$. For graphene, $\varepsilon_{\mathbf{k}}=\hbar v_{\mathrm{F}} k$ with $v_{\mathrm{F}}=10^{6} \mathrm{~m} / \mathrm{s}$ being the Fermi velocity and

$$
\boldsymbol{\Omega}_{\mathbf{k}}=\alpha_{\mathrm{R}}\left(-\sin \theta_{\mathbf{k}}, \cos \theta_{\mathbf{k}}, 0\right)
$$

with $\theta_{\mathbf{k}}$ being the polar angle of momentum $\mathbf{k}$. Therefore in the absence of any external magnetic field

$$
\boldsymbol{\omega}_{\mathbf{k}}=2 \alpha_{\mathrm{R}}\left(-\tan \theta_{\mathbf{k}}, 1,0\right) /\left(\hbar v_{\mathrm{F}}\right),
$$

which depends on the angle $\theta_{\mathbf{k}}$ rather than the magnitude of $k$. This indicates that the inhomogeneous broadening is insensitive to temperature and electron density as long as $\alpha_{\mathrm{R}}$ is fixed. Therefore the spin diffusion is only possible to be modulated effectively by the scattering. ${ }^{42}$ However, in this work it is revealed that when the scattering is strong enough (just as in the graphene layer under study), the spin diffusion becomes insensitive to the scattering. As a result, the spin diffusion is uniquely determined by $\alpha_{\mathrm{R}}$. Moreover, the mean spin precession frequency $\left\langle\boldsymbol{\omega}_{\mathbf{k}}\right\rangle=\frac{2 \alpha_{\mathrm{R}}}{\hbar v_{\mathrm{F}}}(0,1,0)$ shows a strong anisotropy which can also lead to the anisotropy of spin diffusion with respect to the spin polarization direction. This anisotropy is found to be quite different from the widely believed one predicted from the two-component driftdiffusion model. ${ }^{43-47}$ The discrepancy reveals the inadequacy of the two-component drift-diffusion model, especially for the cases with spin precession in spatial domain.

This paper is organized as follows. In Sec. II, we present the model and introduce the KSBEs. In Sec. III, we first investigate the spin relaxation by fitting the experimental data from $\mathrm{Pi}$ et al. ${ }^{31}$ to obtain essential parameters and then study the spin diffusion and transport in graphene. Both the analytical and numerical investigations are performed. By comparing the results from the analytical and numerical studies, we find that the analytical model depicts the zero-electric-field spin diffusion perfectly and the nonzero-electric-field spin transport with a small discrepancy which increases with the strength of the electric field. At last we summarize in Sec. IV.

\section{MODEL AND KSBES}

The $n$-doped graphene monolayer under investigation is on a $\mathrm{SiO}_{2}$ substrate perpendicular to the $z$-axis. The depth of the $\mathrm{SiO}_{2}$ substrate is assumed to be $a=300 \mathrm{~nm}$ and the electric field along the $z$-axis is $E_{z}=V_{g} / a$ with $V_{g}$ being the gate voltage. The spins are injected at $x=0$ and diffuse or transport along the $x$-axis. The external electric field, if applied, is along the $x$-axis, i.e., $\mathbf{E}=$ $E \hat{\mathbf{x}}$. Under the basis laid out in Refs. 25 and 32, the Hamiltonian of electrons can be written as ${ }^{25}$

$$
\begin{aligned}
H= & \sum_{\mu \mathbf{k} s s^{\prime}}\left[\left(\varepsilon_{\mathbf{k}}-\lambda_{\mathrm{I}}+e E x\right) \delta_{s s^{\prime}}+\boldsymbol{\Omega}_{\mathbf{k}} \cdot \boldsymbol{\sigma}_{s s^{\prime}}\right] c_{\mu \mathbf{k} s}{ }^{\dagger} c_{\mu \mathbf{k} s^{\prime}} \\
& +H_{\mathrm{int}} .
\end{aligned}
$$

Here $\mu$ labels the valley located at $K$ or $K^{\prime}$ point, $\boldsymbol{\sigma}$ denote the Pauli matrices and $c_{\mu \mathbf{k} s}\left(c_{\mu \mathbf{k} s}{ }^{\dagger}\right)$ is the annihilation (creation) operator of electron in $\mu$ valley with momentum $\mathbf{k}$ (relative to the valley center) and spin $s\left(s= \pm \frac{1}{2}\right) . \quad \lambda_{\mathrm{I}}$ is the intrinsic spin-orbit coupling constant and $-e$ is the electron charge $(e>0)$. The coefficient in the Rashba term $\boldsymbol{\Omega}_{\mathbf{k}}$ [Eq. (2)] reads $\alpha_{\mathrm{R}}=\zeta E_{z}+\eta$, with the first term contributed by the electric field along the $z$-axis and the second term by the substrate (including the impurities initially present inside) as well as the adatoms from surface chemical doping. The coefficient $\zeta$ is $5 \times 10^{-3} \mathrm{meV} \cdot \mathrm{nm} / \mathrm{V}$ (Refs. 26 and 32). The Hamiltonian $H_{\text {int }}$ consists of the electronimpurity, electron-phonon as well as electron-electron Coulomb interactions. ${ }^{25}$ We adopt the minimal model proposed by Adam and Das Sarma ${ }^{48}$ to depict the electron-impurity scattering. Within this model, only the intravalley electron-impurity scattering is important while the intervalley electron-impurity scattering is negligible due to the large momentum transfer from one valley to the other and the finite distance between the impurity layer and the graphene plane. The intravalley electron-impurity scattering matrix element reads $\left|U_{\mathbf{k}-\mathbf{k}^{\prime}}\right|^{2}=N_{i}\left|V_{\mathbf{k}-\mathbf{k}^{\prime}}\right|^{2} e^{-2 d\left|\mathbf{k}-\mathbf{k}^{\prime}\right|}{ }^{48}$ where $N_{i}$ is the effective impurity density, $d$ is the effective distance of impurities from the graphene layer ${ }^{48}$ and $V_{\mathbf{k}-\mathbf{k}^{\prime}}$ is the Coulomb potential under the random phase approximation. ${ }^{49}$ The electron-phonon scattering includes the intravalley electron-acoustic phonon scattering, ${ }^{50}$ the intervalley and intravalley electron-optical phonon scattering, ${ }^{51}$ as well as the intravalley electron-optical surface phonon scattering. ${ }^{52}$ The electron-electron Coulomb scattering includes both the intervalley and intravalley scattering, with the screening under random phase approximation given in Ref. 49. 
By using the nonequilibrium Green function method, ${ }^{53}$ the KSBEs are constructed $\operatorname{as}^{37}$

$$
\begin{aligned}
\frac{\partial \rho_{\mu \mathbf{k}}(x, t)}{\partial t}= & \left.\frac{\partial \rho_{\mu \mathbf{k}}(x, t)}{\partial t}\right|_{\mathrm{dri}}+\left.\frac{\partial \rho_{\mu \mathbf{k}}(x, t)}{\partial t}\right|_{\mathrm{dif}} \\
& +\left.\frac{\partial \rho_{\mu \mathbf{k}}(x, t)}{\partial t}\right|_{\mathrm{coh}}+\left.\frac{\partial \rho_{\mu \mathbf{k}}(x, t)}{\partial t}\right|_{\text {scat }}
\end{aligned}
$$

Here $\rho_{\mu \mathbf{k}}(x, t)$ represent the density matrices of electrons with relative momentum $\mathbf{k}$ in valley $\mu$ at position $x$ and time $t .\left.\quad \frac{\partial \rho_{\mu \mathbf{k}}(x, t)}{\partial t}\right|_{\mathrm{dri}}=\frac{e E}{\hbar} \frac{\partial \rho_{\mu \mathbf{k}}(x, t)}{\partial k_{x}}$ are the driving terms from the external electric field (the fluctuation of electron density is neglected and thus the total electric field is taken to be the external one). The diffusion terms due to the spatial gradient are

$$
\begin{aligned}
\left.\frac{\partial \rho_{\mu \mathbf{k}}(x, t)}{\partial t}\right|_{\text {dif }} & =-\frac{\partial \varepsilon_{\mathbf{k}}}{\hbar \partial k_{x}} \frac{\partial \rho_{\mu \mathbf{k}}(x, t)}{\partial x} \\
& =-v_{\mathrm{F}} \cos \theta_{\mathbf{k}} \frac{\partial \rho_{\mu \mathbf{k}}(x, t)}{\partial x} .
\end{aligned}
$$

$\left.\frac{\partial \rho_{\mu \mathbf{k}}(x, t)}{\partial t}\right|_{\text {coh }}$ and $\left.\frac{\partial \rho_{\mu \mathbf{k}}(x, t)}{\partial t}\right|_{\text {scat }}$ are the coherent and scattering terms, respectively. Their expressions can be found in Ref. 25. In the steady-state scattering-free spin diffusion, the spatial spin precession frequency, given by Eq. (3), is immediately obtained according to the KSBEs. ${ }^{39-41}$

\section{SPIN RELAXATION AND SPIN DIFFUSION AND TRANSPORT}

In the following, we first study the spin relaxation in graphene by fitting the experimental data from $\mathrm{Pi}$ et $a .^{31}$ to obtain information on impurities (including the effective density as well as the distance from the graphene layer) and the chemical doping dependence of the Rashba spin-orbit coupling coefficient. We then use the information to study the spin diffusion and transport in graphene, first analytically for the case with strong electron-impurity scattering only, and then numerically with all the scattering explicitly included.

\section{A. Spin relaxation time}

We fit the chemical doping dependence of spin relaxation time and diffusion coefficient from $\mathrm{Pi}$ et al. [Fig. 3(c) in Ref. 31] to establish: (i) the density and typical distance from the graphene layer of charged impurities initially present in the substrate and those of the chemical doping adatoms; and (ii) the dependence of $\alpha_{\mathrm{R}}$ on chemical doping. The electron density $N_{e}$ is $2.9 \times 10^{12} \mathrm{~cm}^{-2}$ and the temperature $T$ is $18 \mathrm{~K}^{31}$ The electrons are initially polarized in the $x-y$ plane $^{31}$ with the polarization $P_{0}$ assumed to be 0.05 . To perform the fitting, the KSBEs are solved in the time domain under spatial uniform case, as carried out recently by Zhou and $\mathrm{Wu}$ in the ultraclean graphene monolayer. ${ }^{25}$ (An analytical study of spin relaxation time in graphene is also given in Appendix A.) The diffusion coefficient $D$ given by $\mathrm{Pi}$ et al. is actually for spin instead of charge, although it is treated as the charge diffusion coefficient in the experiment. ${ }^{31}$ In fact, these two coefficients are usually close to each other and Józsa et al. found this most likely to be the case in graphene when the electron density is high $\left(\sim 3 \times 10^{12} \mathrm{~cm}^{-2}\right)^{30}$ due to the weak electron-electron Coulomb scattering. ${ }^{25}$ Therefore we fit the experimental data with the charge diffusion coefficient $D=\frac{\sqrt{\pi N_{e}}}{2 e} \hbar v_{\mathrm{F}} \mu_{e}$, where $\mu_{e}$ is the electron mobility obtained under a small in-plane electric field. ${ }^{25}$

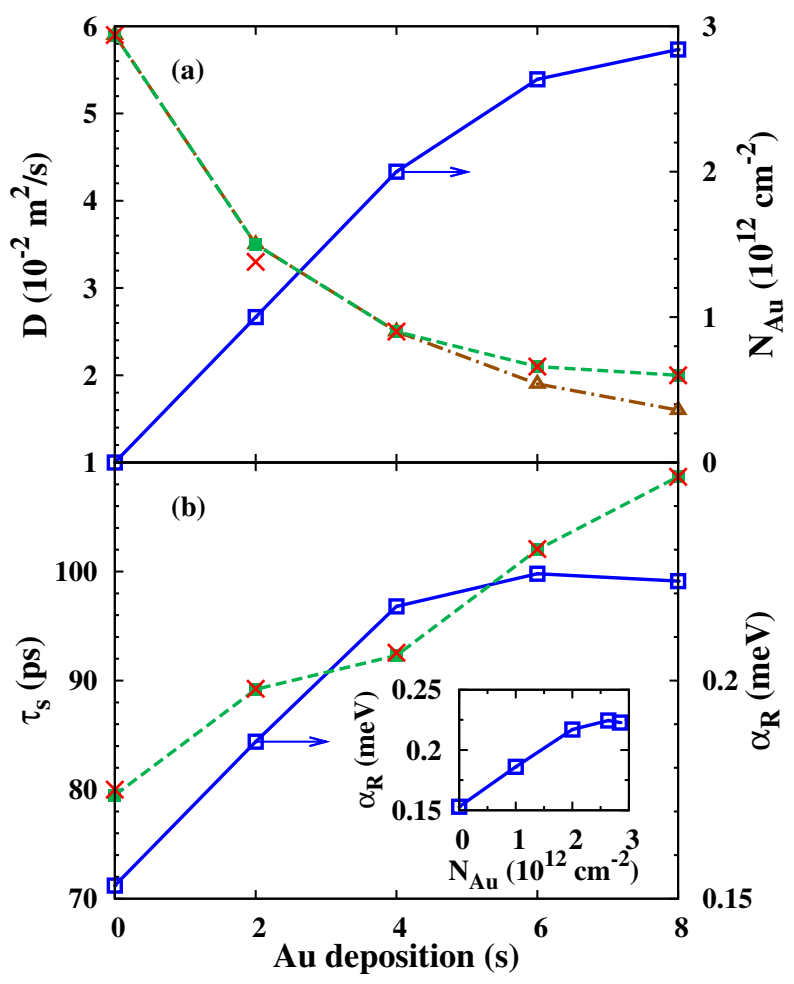

FIG. 1: (Color online) (a) Chain curve with triangles: deposition time dependence of calculated diffusion coefficient, with the $\mathrm{Au}$ density growing linearly with the deposition time with a fixed rate of $5 \times 10^{11}$ atom $/\left(\mathrm{cm}^{2} \cdot \mathrm{s}\right) .{ }^{31}$ Dashed curve with closed squares: deposition time dependence of calculated diffusion coefficient, with the deposition time dependence of $\mathrm{Au}$ density given by the solid curve (the scale is on the righthand side of the frame). Crosses: experimental data from Pi et al.. ${ }^{31}$ (b) Dashed curve with closed squares: deposition time dependence of calculated spin relaxation time, with the deposition time dependence of $\alpha_{\mathrm{R}}$ shown by the solid curve with open squares (the scale is on the right-hand side of the frame). Crosses: experimental data from Pi et al.. ${ }^{31}$ Inset of (b): dependence of $\alpha_{\mathrm{R}}$ on Au density. 
We first make use of the value of $D \approx 0.059 \mathrm{~m}^{2} / \mathrm{s}$ for the case without surface chemical doping given in Ref. 31 to explore the information of impurities initially present in the substrate. This single value of $D$ is not sufficient for us to fix both the effective density and distance from the graphene layer of these impurities. However, these details are not essential and we just choose two proper parameters, e.g., $N_{s}=2.1 \times 10^{12} \mathrm{~cm}^{-2}$ and $d_{s}=0.7 \mathrm{~nm}$, to ensure $D \approx 0.059 \mathrm{~m}^{2} / \mathrm{s}$. The surface chemical doping deposits $\mathrm{Au}$ atoms on the graphene surface with a growth rate of $5 \times 10^{11}$ atom $/\left(\mathrm{cm}^{2} \cdot \mathrm{s}\right) \cdot{ }^{31}$ By fitting the deposition time (adatom density) dependence of $D,{ }^{31}$ the distance of adatoms from the graphene layer $d_{\mathrm{Au}}$ is obtained to be about $0.2 \mathrm{~nm}$. Nevertheless, the fitting does not confirm with the experimental data well when the deposition time exceeds $4 \mathrm{~s}$ [compare the fitting data (chain curve with triangles) to the experimental data (crosses) in Fig. 1(a)]. This indicates that the effective density of adatoms does not increase linearly with time any more when the doping has been performed for several seconds. Therefore, when the doping time is longer than $4 \mathrm{~s}$, we choose the proper density of adatoms to reproduce the experimental diffusion coefficient. In Fig. 1(a), the deposition time dependence of Au density is plotted by the solid curve with open squares (the scale is on the right-hand side of the frame) and that of the calculated diffusion coefficient is shown by the dashed curve with closed squares.

With the parameters for two kinds of impurities obtained, we then fit the spin relaxation time $\tau_{s}$ to obtain $\alpha_{\mathrm{R}}$ under different deposition times. In Fig. 1(b), the deposition time dependence of fitted $\alpha_{\mathrm{R}}$ is shown by the solid curve with open squares (the scale is on the righthand side of the frame) and that of the calculated spin relaxation time is shown by the dashed curve with closed squares. The crosses represent the experimental spin relaxation times under different deposition times. In the inset of Fig. 1(b), we also plot the dependence of $\alpha_{\mathrm{R}}$ on $\mathrm{Au}$ density $N_{\mathrm{Au}}$. It is shown that $\alpha_{\mathrm{R}}$ increases approximately linearly with $\mathrm{Au}$ density when the latter is not so high. The fitted value of $\alpha_{\mathrm{R}}$ is comparable to the value estimated by Ertler et al. when taking account of the adatoms, i.e., $0.3 \mathrm{meV} .^{32}$ It is noted that $\alpha_{\mathrm{R}} \tau_{p} / \hbar$ has the largest value $0.027 \ll 1$ with $\tau_{p}=\sqrt{N_{e} \pi} \frac{\hbar}{e v_{\mathrm{F}}} \mu_{e}=0.12 \mathrm{ps}$ (Ref. 54) when $N_{\mathrm{Au}}=0$. Therefore, the electron system is in the strong scattering limit (the electron-impurity scattering is dominant), let alone when the temperature is increased or the chemical doping is performed. It is necessary to point out that in the experiment the gate voltage $V_{g}$ is adjusted to keep $N_{e}$ constant during chemical doping as adatoms also donate electrons to the graphene layer. ${ }^{31}$ However, $V_{g}$ does not exceed $200 \mathrm{~V}$ and the term $\zeta E_{z}=\zeta V_{g} / a$ is at least two orders of magnitude smaller than $\alpha_{\mathrm{R}}$. Therefore $\alpha_{\mathrm{R}} \approx \eta$ and is solely determined by the impurities. When $N_{\mathrm{Au}}=0, \eta=0.153 \mathrm{meV}$ and is contributed by the impurities in the substrate.

\section{B. Spin diffusion and transport: analytical study}

\section{Spin diffusion}

In this section we study the spin diffusion in graphene analytically for the case with only the electron-impurity scattering. No external electric field is present. We first perform the Fourier transformation of the steady-state KSBEs with respect to the polar angle $\theta_{\mathbf{k}}$ and then retain the equations involving the lowest three orders. ${ }^{41}$ The neglect of the higher orders will not lose much information in the strong scattering limit where the electron distribution approaches isotropy in the momentum space. As a result the following second-order differential equation about $\rho_{\mu k}^{0}(x)\left[\rho_{\mu k}^{l}(x)=\frac{1}{2 \pi} \int_{0}^{2 \pi} d \theta_{\mathbf{k}} \rho_{\mu \mathbf{k}}(x) e^{-i l \theta_{\mathbf{k}}}\right.$ and $\left.\rho_{\mu \mathbf{k}}(x) \equiv \rho_{\mu \mathbf{k}}(x,+\infty)\right]$ is obtained:

$$
\begin{aligned}
& \partial_{x}^{2} \rho_{\mu k}^{0}(x)+i \frac{2 \alpha_{\mathrm{R}}}{\hbar v_{\mathrm{F}}}\left[\sigma_{y}, \partial_{x} \rho_{\mu k}^{0}(x)\right]-\frac{\alpha_{\mathrm{R}}^{2}}{\hbar^{2} v_{\mathrm{F}}^{2}}\left[\sigma_{x},\left[\sigma_{x}, \rho_{\mu k}^{0}(x)\right]\right] \\
& -\frac{\alpha_{\mathrm{R}}^{2}}{\hbar^{2} v_{\mathrm{F}}^{2}}\left[\sigma_{y},\left[\sigma_{y}, \rho_{\mu k}^{0}(x)\right]\right]=0 .
\end{aligned}
$$

It is noted that with only the lowest three orders of $\rho_{\mu k}^{l}(x)$ considered from the beginning, the electron-impurity scattering time is actually absent from the above equation (refer to Appendix B for detail). This indicates that in the strong scattering limit the spin diffusion becomes insensitive to scattering in graphene. We define the "spin vector" as $\mathbf{S}_{\mu k}^{0}(x)=\operatorname{Tr}\left[\rho_{k}^{0}(x) \boldsymbol{\sigma}\right]$ and $\mathbf{S}_{\mu k}^{0}(x)$ can be solved from Eq. (7) with boundary conditions (refer to Appendix B for detail). Then one can calculate the total spin signal contributed by all the different electron states in two valleys as

$$
\begin{aligned}
\mathbf{S}(x) & =\frac{1}{4 \pi^{2}} \sum_{\mu} \int_{0}^{+\infty} d k \int_{0}^{2 \pi} d \theta_{\mathbf{k}} k \operatorname{Tr}\left[\rho_{\mathbf{k}}(x) \boldsymbol{\sigma}\right] \\
& =\frac{1}{\pi} \int_{0}^{+\infty} d k k \mathbf{S}_{\mu k}^{0}(x) .
\end{aligned}
$$

In the following we present the solutions of $\mathbf{S}(x)$ under three typical boundary conditions.

For boundary condition (I) $\mathbf{S}_{\mu k}^{0}(0)=\left(S_{\mu k}^{0}(0), 0,0\right)$ and $\mathbf{S}_{\mu k}^{0}(+\infty)=0$, which corresponds to the case with the injected spins polarized along the $x$-axis,

$$
\mathbf{S}(x)=S(0) e^{-x / l_{x}}\left(\begin{array}{c}
\sqrt{1+\Delta^{2}} \sin (\omega x+\phi) \\
0 \\
c_{1} \sin (\omega x)
\end{array}\right) \text {. }
$$

For boundary condition (II) $\mathbf{S}_{\mu k}^{0}(0)=\left(0, S_{\mu k}^{0}(0), 0\right)$ and $\mathbf{S}_{\mu k}^{0}(+\infty)=0$,

$$
\mathbf{S}(x)=S(0) e^{-x / l_{y}}\left(\begin{array}{l}
0 \\
1 \\
0
\end{array}\right)
$$


For boundary condition (III) $\mathbf{S}_{\mu k}^{0}(0)=\left(0,0, S_{\mu k}^{0}(0)\right)$ and $\mathbf{S}_{\mu k}^{0}(+\infty)=0$,

$$
\mathbf{S}(x)=S(0) e^{-x / l_{z}}\left(\begin{array}{c}
c_{2} \sin (\omega x) \\
0 \\
-\sqrt{1+\Delta^{2}} \sin (\omega x-\phi)
\end{array}\right) .
$$

In the above equations

$$
S(0)=\frac{1}{\pi} \int_{0}^{+\infty} d k k S_{\mu k}^{0}(0)
$$

and

$$
\begin{aligned}
& l_{x}=l_{z}=\frac{\sqrt{7}}{(2 \sqrt{2}-1) \sqrt{1+2 \sqrt{2}}} \frac{\hbar v_{\mathrm{F}}}{\alpha_{\mathrm{R}}}, \\
& l_{y}=\frac{\hbar v_{\mathrm{F}}}{2 \alpha_{\mathrm{R}}}, \\
& \omega=\sqrt{1+2 \sqrt{2}} \frac{\alpha_{\mathrm{R}}}{\hbar v_{\mathrm{F}}} .
\end{aligned}
$$

$c_{1}=-\frac{4}{(1+\sqrt{2}) \sqrt{1+2 \sqrt{2}}}, \quad c_{2}=\frac{(20 \sqrt{2}-24) \sqrt{1+2 \sqrt{2}}}{7}, \Delta=$ $\frac{8 \sqrt{2}-11}{\sqrt{7}}$ and $\phi=\arctan \frac{1}{\Delta}$. It is noted that the spin precession frequency given by the simplified model is $\omega \approx 1.96 \frac{\alpha_{\mathrm{R}}}{\hbar v_{\mathrm{F}}}$, a little smaller than $\left|\left\langle\boldsymbol{\omega}_{\mathbf{k}}\right\rangle\right|=\frac{2 \alpha_{\mathrm{R}}}{\hbar v_{\mathrm{F}}}$ due to the approximations made here.

From Eqs. (9)-(11) one notices that in the strong scattering limit, the spin diffusion is not only insensitive to the scattering, but also unrelated to temperature $T$ and electron density $N_{e}$. Nevertheless, the coefficient $\alpha_{\mathrm{R}}$ may depend on $T$ and/or $N_{e}$, with the relation unclear so far. For simplicity we assume $\alpha_{\mathrm{R}}$ to be independent of $T$ and $N_{e}$ in this work. As a result, the spin diffusion in the strong scattering limit is uniquely determined by $\alpha_{\mathrm{R}}$, which is only modulated by chemical doping. Eqs. (9)(11) indicate a strong anisotropy of spin diffusion with respect to the spin-polarization direction. For the cases with the injected spins polarized along the $x$ - and $z$-axis, both the spin signals show an exponential decay in the magnitude accompanying with the precession in the $x$ $z$ plane. The spin precessions have the same frequency $\omega$ except for a phase difference. However, when the injected spins are polarized along the $y$-axis, the spin signal decays exponentially without any precession, i.e., it is bound along the $y$-axis. The above phenomena are understood by noticing that the mean effective magnetic field felt by the diffusing electrons is along the $y$-axis as $\left\langle\boldsymbol{\omega}_{\mathbf{k}}\right\rangle=\frac{2 \alpha_{\mathrm{R}}}{\hbar v_{\mathrm{F}}}(0,1,0)$. In the non-local spin valve experiments, the spin diffusion length is usually measured from the exponential decay of spin signal with the increasing spacing between the central spin-injector and -detector ferromagnetic electrodes. ${ }^{27,28}$ In these experiments, the ferromagnetic electrodes happen to be magnetized along the $y$-axis and therefore the injected and detected spin polarizations are both along the $y$-axis. With such configuration, the exponential decay of spin signal with increasing spacing between the electrodes can be well observed. However, if the injected spins are polarized in the $x-z$ plane, the spatial spin precession is expected to be detected.

Besides the anisotropy of spin precession, the spin diffusion length also shows an anisotropy as

$$
l_{x}=l_{z} \approx 1.48 l_{y}
$$

with $l_{y}=\hbar v_{\mathrm{F}} /\left(2 \alpha_{\mathrm{R}}\right)$. In fact, when the injected spins are polarized along any other direction in the $x-z$ plane, the spin diffusion length is all the same as $l_{x}\left(l_{z}\right)$ [for this case the solution of $\mathbf{S}(x)$ is the combination of Eqs. (9) and (11)]. However, based on the widely utilized twocomponent drift-diffusion model ${ }^{43-47}$ which gives $l_{s}=$ $\sqrt{D \tau_{s}}$ [Eq. (D11) in Appendix D], one may expect that the spin diffusion lengths satisfy

$$
l_{x}=l_{y}=\sqrt{2} l_{z}=\hbar v_{\mathrm{F}} /\left(2 \alpha_{\mathrm{R}}\right)
$$

as the spin relaxation times in time domain follow (refer to Appendix A)

$$
\tau_{x}=\tau_{y}=2 \tau_{z}=\hbar^{2} /\left(2 \alpha_{\mathrm{R}}^{2} \tau_{p}\right)
$$

and $D=v_{\mathrm{F}}^{2} \tau_{p} / 2$. It is noted that only when the injected spins are polarized along the $y$-axis, for which no spin precession exists, the two-component drift-diffusion model gives the result in consistence with that from the KSBEs, i.e.,

$$
l_{y}=\hbar v_{\mathrm{F}} /\left(2 \alpha_{\mathrm{R}}\right) .
$$

The discrepancy in the anisotropies given by the KSBEs and the two-component drift-diffusion model strongly indicates the inadequacy of the two-component driftdiffusion model. Due to the loss of the off-diagonal spin components, i.e., the spin coherence, the two-component drift-diffusion model not only fails to predict the spin precession in spatial domain in the absence of an external magnetic field, but also incorrectly inherits the anisotropy from the spin relaxation in time domain. We emphasize that the reason for the different anisotropic properties of spin diffusion in spatial domain and spin relaxation in time domain is that the inhomogeneous broadening is quite different in these two cases. In spatial domain the inhomogeneous broadening governing the spin diffusion arises from the $\mathbf{k}$ dependence of $\boldsymbol{\omega}_{\mathbf{k}}$, while in time domain from that of $\boldsymbol{\Omega}_{\mathbf{k}}$. Popinciuc et al. reported the relationship between the in-plane and out-ofplane spin relaxation times directly from the anisotropy of spin diffusion via the two-component drift-diffusion model. ${ }^{28}$ However, based on the above discussion, one may realize that studying the anisotropy of spin relaxation time in such a way can be incorrect. Finally, from another point of view, if the two-component driftdiffusion model is still used, then in order to reflect the correct anisotropy of spin diffusion, the spin diffusion coefficient has to differ from the charge diffusion coefficient and shows an anisotropy as $D_{x}=0.5 D_{z} \approx 2.2 D_{y}$ with $D_{y}=v_{\mathrm{F}}^{2} \tau_{p} / 2$. 
It should be pointed out that all the above analysis and conclusion also apply to the electron system where the energy spectrum is parabolic in momentum and the linear Rashba spin-orbit coupling term $\boldsymbol{\Omega}_{\mathbf{k}} \propto$ $k\left(-\sin \theta_{\mathbf{k}}, \cos \theta_{\mathbf{k}}, 0\right)$ is dominant, such as that in the asymmetric InAs quantum wells. ${ }^{42}$ That is because the steady-state scattering-free spatial spin precession frequency $\boldsymbol{\omega}_{\mathrm{k}}$ in this system has the similar momentum dependence as shown in Eq. (3). ${ }^{42}$ However, for electron system in the absence of the DP term but under a magnetic field perpendicular to both the spin polarization and spin transport directions such as in bulk silicon ${ }^{55}$ and symmetric silicon quantum wells, ${ }^{41}$ or with the Dresselhaus term ${ }^{56}$ containing the cubic dependence on momentum such as in GaAs quantum wells, ${ }^{39,40}$ the situation is quite different as $\boldsymbol{\omega}_{\mathbf{k}}$ depends on the magnitude of momentum. In fact, it has been revealed in the symmetric silicon quantum wells under an in-plane magnetic field that the scattering can suppress spin diffusion effectively in the strong scattering limit. ${ }^{41}$

\section{Spin transport}

We further take account of the electric field along the $x$-axis to study the spin transport. Still only the strong electron-impurity scattering is included. The secondorder differential equation about $\rho_{\mu k}^{0}(x)$, corresponding to Eq. (7) but including the driving term, reads (refer to Appendix C)

$$
\begin{aligned}
& \partial_{x}^{2} \rho_{\mu k}^{0}(x)+i \frac{2 \alpha_{\mathrm{R}}}{\hbar v_{\mathrm{F}}}\left[\sigma_{y}, \partial_{x} \rho_{\mu k}^{0}(x)\right]-\frac{\alpha_{\mathrm{R}}^{2}}{\hbar^{2} v_{\mathrm{F}}^{2}}\left[\sigma_{x},\left[\sigma_{x}, \rho_{\mu k}^{0}(x)\right]\right] \\
& -\frac{\alpha_{\mathrm{R}}^{2}}{\hbar^{2} v_{\mathrm{F}}^{2}}\left[\sigma_{y},\left[\sigma_{y}, \rho_{\mu k}^{0}(x)\right]\right]-e E \partial_{x} \partial_{\varepsilon_{\mathbf{k}}} \rho_{\mu k}^{0}(x) \\
& -i \frac{\alpha_{\mathrm{R}} e E}{\hbar v_{\mathrm{F}}}\left[\sigma_{y}, \partial_{\varepsilon_{\mathbf{k}}} \rho_{\mu k}^{0}(x)\right]=0
\end{aligned}
$$

It should be pointed out that when the electric field is so large that the electron density matrices $\rho_{\mu \mathbf{k}}(x,+\infty)$ become strongly anisotropic due to the driving of the electric field, retaining only the lowest three orders of $\rho_{\mu k}^{l}(x)$ to obtain the above equation of $\rho_{\mu k}^{0}(x)$ may not be sufficient. The second-order differential equation about $\mathbf{S}_{\mu k}^{0}(x)$ is obtained from the above equation and that about $\mathbf{S}(x)$ can be obtained by further summing over $\mathbf{k}$ and $\mu$ (refer to Appendix C). With the same three different typical boundary conditions presented in the previous section, $\mathbf{S}(x)$ is solved to have the same form as Eqs. (9)(11) except that the parameters are now electric-field de- pendent. Explicitly,

$$
\begin{aligned}
& l_{x}^{\prime}=l_{z}^{\prime}=\frac{1}{\mathscr{E} / 2+F(\mathscr{E})} \frac{\hbar v_{\mathrm{F}}}{\alpha_{\mathrm{R}}}, \\
& l_{y}^{\prime}=\frac{1}{\mathscr{E} / 2+\sqrt{4+\mathscr{E}^{2} / 4}} \frac{\hbar v_{\mathrm{F}}}{\alpha_{\mathrm{R}}}, \\
& \omega^{\prime}=G(\mathscr{E}) \frac{\alpha_{\mathrm{R}}}{\hbar v_{\mathrm{F}}}, \\
& c_{1}^{\prime}=-\frac{1}{2} \frac{\sqrt{\mathscr{E}^{4}+48 \mathscr{E}^{2}+512}}{\sqrt{\mathscr{E}^{2}+7} F(\mathscr{E})+5 G(\mathscr{E})}, \quad c_{2}^{\prime}=\frac{1}{2} \frac{\sqrt{\mathscr{E}^{4}+48 \mathscr{E}^{2}+512}}{\sqrt{\mathscr{E}^{2}+7} F(\mathscr{E})+3 G(\mathscr{E})}, \\
& \Delta^{\prime}=\frac{5 F(\mathscr{E})-\sqrt{\mathscr{E}^{2}+7} G(\mathscr{E})}{\sqrt{\mathscr{E}^{2}+7} F(\mathscr{E})+5 G(\mathscr{E})} \text { and } \phi^{\prime}=\arctan \frac{1}{\Delta^{\prime}} \text {. In the above } \\
& \text { equations, } \\
& \begin{aligned}
\mathscr{E}= & \frac{e E}{S(0) \pi \alpha_{\mathrm{R}} \beta \hbar v_{\mathrm{F}}} \ln \frac{1+e^{\beta \mu_{\uparrow}}}{1+e^{\beta \mu_{\downarrow}}} \\
F(\mathscr{E})= & \frac{\sqrt{\mathscr{E}^{4}+48 \mathscr{E}^{2}+512}+\mathscr{E}^{2}-8}{16 \sqrt{2} \sqrt{\mathscr{E}^{2}+7}} \\
& \times \sqrt{\sqrt{\mathscr{E}^{4}+48 \mathscr{E}^{2}+512}-\mathscr{E}^{2}+8} \\
G(\mathscr{E})= & \sqrt{1-\mathscr{E}^{2} / 8+\sqrt{\mathscr{E}^{4}+48 \mathscr{E}^{2}+512} / 8}
\end{aligned}
\end{aligned}
$$

Here $\beta=1 /\left(k_{B} T\right)$ and $\mu_{\uparrow}\left(\mu_{\downarrow}\right)$ is the chemical potential of electrons with spin parallel (antiparallel) to the spinpolarization direction. It is noted that when the electric field is absent, i.e., $\mathscr{E}=0$, all the above solutions recover those presented in the previous section.

In most conditions (such as in the present work) electrons in graphene are highly degenerate. In the degenerate limit with small spin polarization, $\mathscr{E} \approx \frac{e E}{\alpha_{\mathrm{R}} k_{\mathrm{F}}}$, where $k_{\mathrm{F}}=\sqrt{\pi N_{e}}$ is the magnitude of the Fermi momentum of unpolarized electrons with density being $N_{e}$ (Appendix C). Differing from the spin diffusion without electric field, the spin transport becomes sensitive to electron density as $\mathscr{E}$ depends on the electron density. In the nondegenerate limit, $\mathscr{E} \approx \frac{e E \beta \hbar v_{\mathrm{F}}}{\alpha_{\mathrm{R}}}$ (Appendix C) and the spin transport becomes sensitive to temperature rather than electron density. Moreover, with this value of $\mathscr{E}$, Eq. (22) becomes

$$
l_{y}^{\prime}=\left[e E \beta / 2+\sqrt{e^{2} E^{2} \beta^{2} / 4+1 / l_{y}^{2}}\right]^{-1},
$$

where $l_{y}$ is the spin diffusion length without electric field [Eq. (14)]. This result recovers that from the twocomponent drift-diffusion model, which apparently fails to correctly reflect the anisotropy of spin transport. ${ }^{13,44}$ Therefore, our investigation again indicates that only when the spatial spin precession is absent, the twocomponent drift-diffusion model gives the appropriate depiction of spin transport.

In Fig. 2 we plot the dependence of $l_{x, y, z}^{\prime}, \omega^{\prime}$ and $\phi^{\prime}$ on $\mathscr{E}$. From Fig. 2(a), one notices that the spin transport length decreases with increasing $\mathscr{E}\left(\mathscr{E} \approx \frac{e E}{\alpha_{\mathrm{R}} k_{\mathrm{F}}}\right)$. On one hand, this means that when the electron density is fixed (e.g., $N_{e}=10^{12} \mathrm{~cm}^{-2}$, for which the variation of $\mathscr{E}$ from -8 to 8 corresponds to a variation of $E$ from about 
-2.2 to $2.2 \mathrm{kV} / \mathrm{cm}$ ), the spin transport is suppressed (enhanced) by increasing the electric field parallel (antiparallel) to the spin injection direction. On the other hand, this also means that when the non-zero electric field parallel (antiparallel) to the spin injection direction is fixed, the spin transport is enhanced (suppressed) by increasing electron density. Fig. 2(b) and (c) indicate that the spin precession frequency $\omega^{\prime}$ and the phase angle $\phi^{\prime}$ vary with $\mathscr{E}$ marginally (with a variation $\sim 2 \%$ ). In fact, when $|\mathscr{E}|$ becomes even larger, both $\omega^{\prime}$ and $\phi^{\prime}$ quickly saturate $\left(\omega^{\prime}\right.$ approaches $\frac{2 \alpha_{R}}{\hbar v_{F}}$ and $\phi^{\prime}$ approaches $\left.\pi / 2\right)$. Therefore, the spin precession pattern in spatial domain is insensitive to the electric field or the electron density.

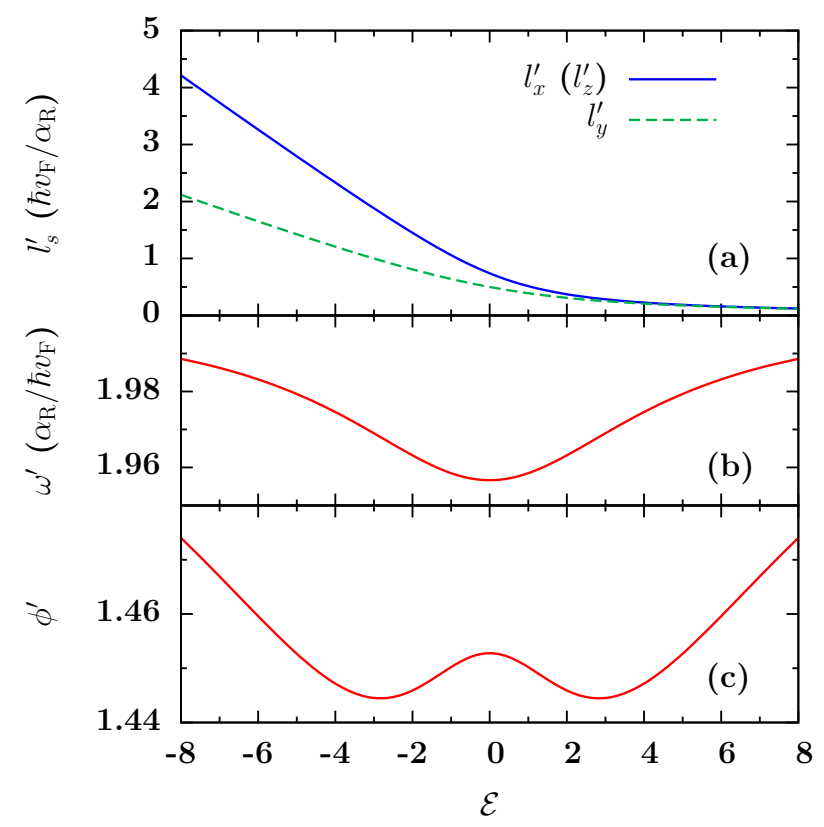

FIG. 2: (Color online) The dependence of (a) spin transport length $l_{x, y, z}^{\prime}$, (b) spin precession frequency $\omega^{\prime}$ and (c) phase angle $\phi^{\prime}$ on $\mathscr{E}$.

\section{Spin diffusion and transport: numerical results}

The KSBEs need to be solved numerically in order to take full account of all the different kinds of scattering as well as the large electric field. To numerically solve the KSBEs, the initial conditions are set as

$$
\begin{aligned}
& \rho_{\mu \mathbf{k}}(0,0)=\frac{F_{\mathbf{k} \uparrow}^{0}+F_{\mathbf{k} \downarrow}^{0}}{2}+\frac{F_{\mathbf{k} \uparrow}^{0}-F_{\mathbf{k} \downarrow}^{0}}{2} \hat{\mathbf{n}} \cdot \boldsymbol{\sigma}, \\
& \rho_{\mu \mathbf{k}}(x>0,0)=\frac{F_{\mathbf{k} \uparrow}^{L}+F_{\mathbf{k} \downarrow}^{L}}{2}, \\
& \sum_{\mu \mathbf{k}} \operatorname{Tr}\left[\rho_{\mu \mathbf{k}}(0,0) \hat{\mathbf{n}} \cdot \boldsymbol{\sigma}\right] / \sum_{\mu \mathbf{k}} \operatorname{Tr}\left[\rho_{\mu \mathbf{k}}(0,0)\right]=P_{0},
\end{aligned}
$$

and the two-side injection boundary conditions ${ }^{39,40}$ are

$$
\begin{aligned}
\left.\rho_{\mu \mathbf{k}}(0, t)\right|_{k_{x}>0} & =\frac{F_{\mathbf{k} \uparrow}^{0}+F_{\mathbf{k} \downarrow}^{0}}{2}+\frac{F_{\mathbf{k} \uparrow}^{0}-F_{\mathbf{k} \downarrow}^{0}}{2} \hat{\mathbf{n}} \cdot \boldsymbol{\sigma}, \\
\left.\rho_{\mu \mathbf{k}}(L, t)\right|_{k_{x}<0} & =\frac{F_{\mathbf{k} \uparrow}^{L}+F_{\mathbf{k} \downarrow}^{L}}{2} .
\end{aligned}
$$

Here the injected spins at left boundary $x=0$ are assumed to be polarized along $\hat{\mathbf{n}}$ with polarization $P_{0}=$ 0.05. $x=L$ stands for the right boundary with $L$ much longer than the spin diffusion or transport length. $F_{\mathbf{k} \uparrow, \downarrow}^{0, L}$ are the Fermi distributions of electrons at the two boundaries when the external electric field is absent. When the electric field is present, $F_{\mathbf{k} \uparrow, \downarrow}^{0, L}$ then stand for the drifted Fermi distributions of hot electrons. ${ }^{25}$ In the previous analytical study the boundary conditions are in fact approximated as the single-side injection case. This approximation works well when the scattering is strong. ${ }^{39}$ By numerically solving the KSBEs, the steady-state distribution of spin polarization along $\hat{\mathbf{n}}$ is obtained as $P(x)=\sum_{\mu \mathbf{k}} \operatorname{Tr}\left[\rho_{\mu \mathbf{k}}(x,+\infty) \hat{\mathbf{n}} \cdot \boldsymbol{\sigma}\right] / \sum_{\mu \mathbf{k}} \operatorname{Tr}\left[\rho_{\mu \mathbf{k}}(x,+\infty)\right]$ and then the spin diffusion or transport length is determined from the exponential decay of $P(x)$ (or its envelope) along the $x$-axis.

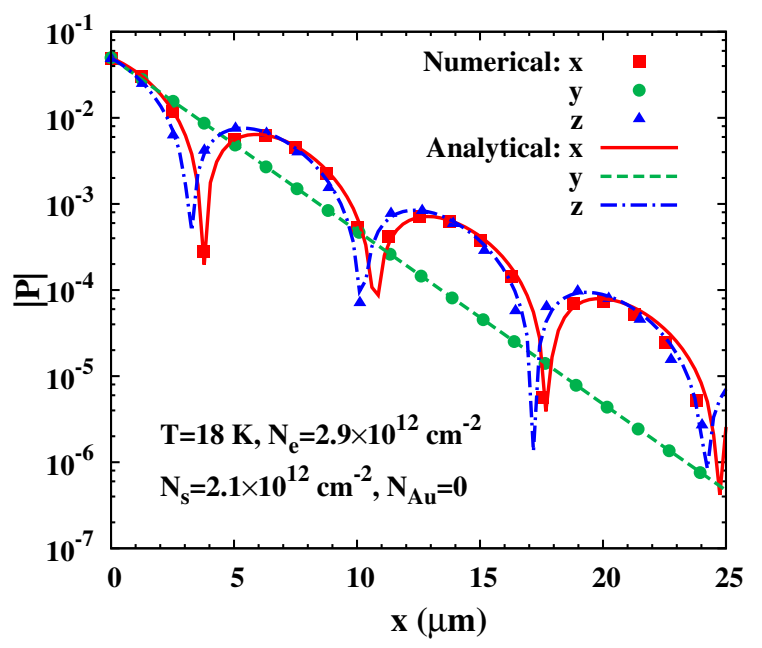

FIG. 3: (Color online) The absolute value of steady-state spin polarization $|P|$ versus position $x$ with the injected spins polarized along the $x$-, $y$ - and $z$-axis, respectively. The squares, circles and triangles are obtained by numerically solving the KSBEs with $T=18 \mathrm{~K}, N_{e}=2.9 \times 10^{12} \mathrm{~cm}^{-2}$, $N_{s}=2.1 \times 10^{12} \mathrm{~cm}^{-2}$ and $N_{\mathrm{Au}}=0$. The solid, dashed and chain curves are calculated from Eqs. (9)-(11) with $P(x)=$ $S_{x}(x) / N_{e}, S_{y}(x) / N_{e}$ and $S_{z}(x) / N_{e}$, respectively.

\section{Anisotropic spin diffusion}

As revealed by the analytical model, the spin diffusion shows anisotropic properties with respect to the polarization direction of injected spins. In Fig. 3, we show the 
spatial distribution of the absolute value of the steadystate spin polarization $|P|$ for the cases with the injected spins polarized along the $x$-, $y$ - and $z$-axis, respectively. $N_{\mathrm{Au}}=0$, with which $\alpha_{\mathrm{R}}=0.153 \mathrm{meV}$. The squares, circles and triangles are obtained by numerically solving the KSBEs while the solid, dashed and chain curves are calculated by Eqs. (9)-(11). When $\alpha_{\mathrm{R}}=0.153 \mathrm{meV}$ the analytical model gives $l_{x}=l_{z} \approx 3.18 \mu \mathrm{m}, l_{y} \approx 2.16 \mu \mathrm{m}$ and $\omega \approx 0.45 \mu \mathrm{m}^{-1}$. The anisotropy of spin diffusion is clearly shown in this figure. It is noted that the simplified analytical model almost perfectly recovers the numerical results [except that the spin precession frequencies for both cases with the injected spins polarized along the $x$ and $z$-axis are numerically shown to be closer to $2 \frac{\alpha_{R}}{\hbar v_{F}}$ rather than $1.96 \frac{\alpha_{\mathrm{R}}}{\hbar v_{\mathrm{F}}}$ given by the analytical study (the difference is expected from the approximations made in the analytical analysis)]. In fact, further numerical calculations show that varying $T$ from 18 to $300 \mathrm{~K}$ and/or $N_{e}$ from 0.5 to $2.9 \times 10^{12} \mathrm{~cm}^{-2}$ changes the numerical results marginally. This is consistent with the conclusion from the analytical model, i.e., the spin diffusion of electrons in graphene is insensitive to $T$ and $N_{e}$ in the strong scattering limit. As a result, in the strong scattering limit, one can depict the spin diffusion quite well with the single parameter $\alpha_{\mathrm{R}}$ via Eqs. (9)-(11).

\section{Chemical doping dependence of spin diffusion}

In Fig. 4, we plot the deposition time dependence of spin diffusion length with $\hat{\mathbf{n}}=\hat{\mathbf{x}}, \hat{\mathbf{y}}$ and $\hat{\mathbf{z}}$ respectively by the solid curves. The spin diffusion lengths are directly obtained from Eqs. (13)-(14). It is shown that with the increase of chemical doping time, $\alpha_{\mathrm{R}}$ increases and the spin diffusion length decreases. For comparison, we also plot the deposition time dependence of spin diffusion length given by the two-component drift-diffusion model (chain curves), i.e., $l_{x}=l_{y}=\sqrt{2} l_{z}=\sqrt{D \tau_{x}}$, with $D$ and $\tau_{x}$ given in Fig. 1. The comparison between these two sets of results shows that, only when the injected spins are polarized along the $y$-axis, the two-component drift-diffusion model yields the same result as that from the KSBEs, just as revealed in the analytical study [refer to Eq. (19) and the discussion there].

\section{Effect of scattering on spin diffusion}

The electron system under investigation is always in the strong scattering limit and therefore the spin diffusion becomes insensitive to scattering. However, the properties of spin diffusion in the weak scattering limit can be different. In order to investigate the spin diffusion with scattering strength ranging from the weak to strong scattering limit, we artificially vary the impurity density in the substrate from 0 to $10^{12} \mathrm{~cm}^{-2}$. At the same time, the chemical doping is absent (no adatom) and $\alpha_{\mathrm{R}}$ is kept as a constant, e.g., $0.153 \mathrm{meV}$. We choose $T=50 \mathrm{~K}$,

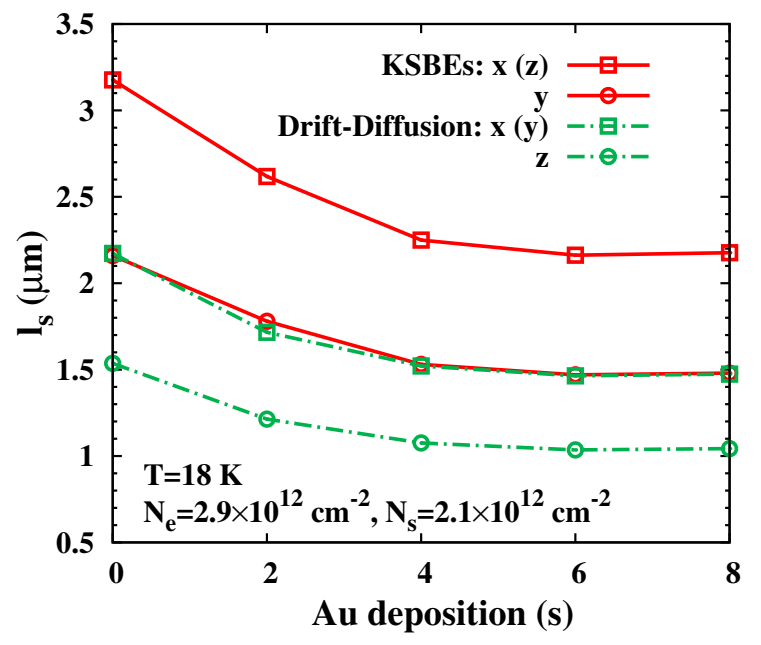

FIG. 4: (Color online) Deposition time dependence of spin diffusion length with the injected spins polarized along the $x$-, $y$ - and $z$-axis, respectively. The results from the KSBEs (solid curves) and the two-component drift-diffusion model (chain curves) are both plotted for comparison.

$N_{e}=5 \times 10^{11} \mathrm{~cm}^{-2}$ and $\hat{\mathbf{n}}=\hat{\mathbf{y}}$. In Fig. 5 we plot the dependence of spin diffusion length $l_{y}$ on the impurity density by the dashed curve. For comparison, we also plot the corresponding dependence of spin relaxation time $\tau_{y}$ on the impurity density by the solid curve (the scale is on the right-hand side of the frame). It is seen that with the increase in $N_{s}$, while $l_{y}$ decreases obviously in the weak scattering limit $\left(N_{s} \lesssim 0.05 \times 10^{12} \mathrm{~cm}^{-2}\right)$ and then saturates in the strong scattering limit, $\tau_{y}$ first decreases in the weak scattering limit (refer to the inset for detail) and then increases almost linearly in the strong scattering limit. ${ }^{37}$ The two-component drift-diffusion model is able to capture the dependence of spin diffusion length on $N_{s}$ by means of the relation $l_{y}=\sqrt{D \tau_{y}}$ : while $D \propto \tau_{p} \propto 1 / N_{s}, \tau_{y}$ decreases with $N_{s}$ in the weak scattering limit and $\propto N_{s}$ in the strong scattering limit; therefore $l_{y}$ first decreases with $N_{s}$ and then becomes insensitive to $N_{s}$ (the insensitivity of $l_{y}$ to $N_{s}$ in the strong scattering limit is revealed previously by the analytical study). It should be emphasized that in Fig. 5 the results are shown with $\alpha_{\mathrm{R}}$ being a constant. In reality, when one further takes account of the increase of $\alpha_{\mathrm{R}}$ with increasing $N_{s}, l_{y}$ should always decrease with increasing $N_{s}$, from the weak scattering limit to the strong scattering limit.

\section{Spin transport under the electric field}

At last we investigate the spin transport under an electric field along the $x$-axis. $T=300 \mathrm{~K}, N_{e}=10^{12} \mathrm{~cm}^{-2}$, $N_{s}=2.1 \times 10^{12} \mathrm{~cm}^{-2}$ and $N_{\mathrm{Au}}=0$. The injected 


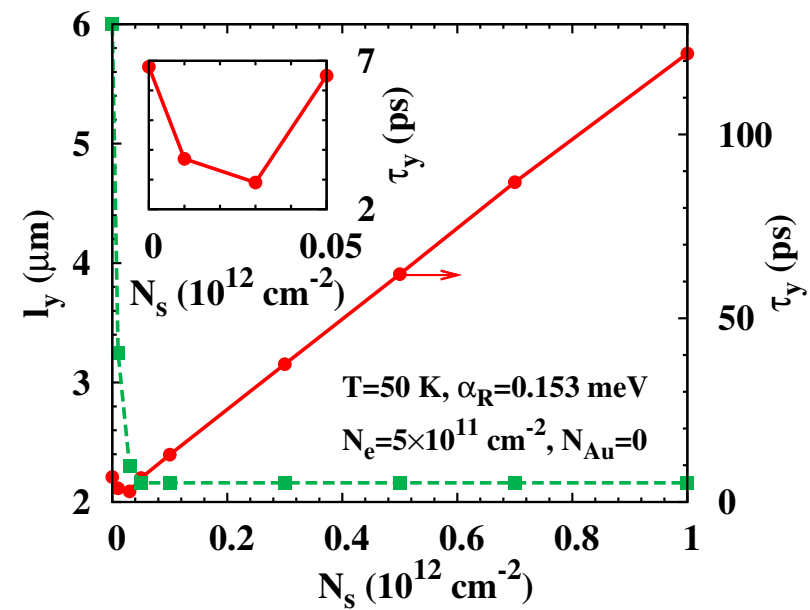

FIG. 5: (Color online) The impurity (in the substrate) density dependence of spin diffusion length (dashed curve) and spin relaxation time (solid curve with the scale on the right-hand side of the frame). The inset shows the detail of the solid curve in the small density regime. The injected spins are polarized along the $y$-axis. $T=50 \mathrm{~K}, N_{e}=5 \times 10^{11} \mathrm{~cm}^{-2}$ and $\alpha_{\mathrm{R}}=0.153 \mathrm{meV}$.

spins are polarized along the $z$-axis. In Fig. 6 the position dependence of $|P|$ under different electric fields as well as the $E$ dependence of $l_{z}$ (squares with the scale on the right-hand side and top of the frame) are plotted. It is shown that while the spin-precession pattern almost keeps the same with varying $E$, the spin transport length is increased (decreased) by increasing the electric field along the $-x(x)$-direction. ${ }^{39,44}$ These results are in consistence with the analytical study presented in Sec. III B 2. For comparison, we further plot the $E$ dependence of $l_{z}$ from Eq. (21) by the double-dotted chain curve with the scale also on the right-hand side and the top of the frame. It is shown that the analytical model depicts the spin transport in the low electric-field regime well except when the electric field antiparallel to the spin-injection direction is large (e.g, a discrepancy reaches $20 \%$ when $E$ reaches $-2 \mathrm{kV} / \mathrm{cm})$.

The electron density dependence of spin transport is also investigated. In Fig. 7, we plot the density dependence of spin transport length under the electric field parallel $(E=0.3 \mathrm{kV} / \mathrm{cm})$ and antiparallel $(E=$ $-0.3 \mathrm{kV} / \mathrm{cm}$ ) to the spin transport direction in (a) and (b), respectively. The squares are from the numerical calculation and the curves are from Eq. (21). It is clearly shown that for the cases with opposite directions of the electric field, the density dependences of spin transport length have opposite tendencies.

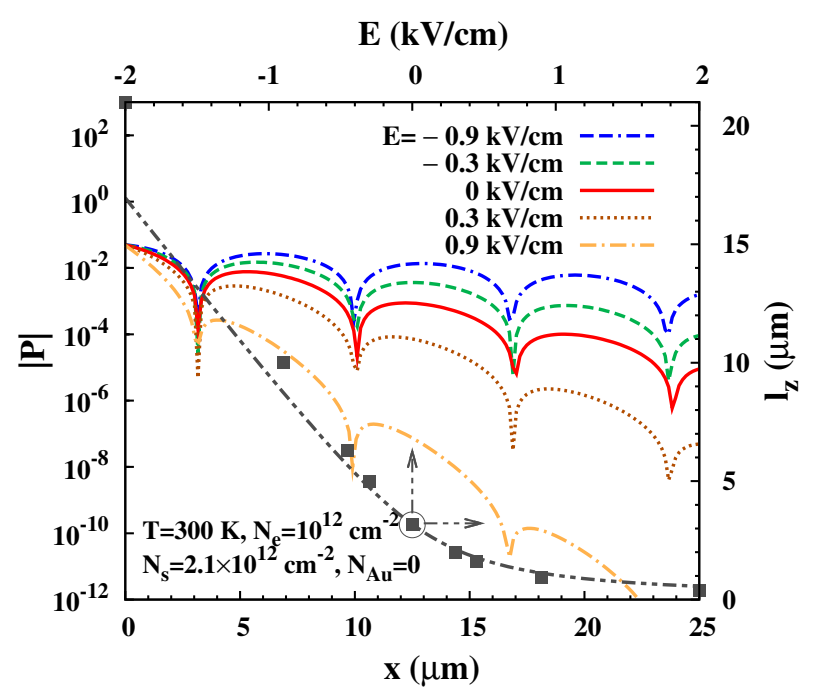

FIG. 6: (Color online) The absolute value of the steady-state spin polarization $|P|$ versus position $x$ under different electric fields. The electric field dependence of spin transport length $l_{z}$ is also plotted with the scale on the right-hand side and top of the frame, where the squares and double-dotted chain curve are obtained from the numerical calculation and from Eq. (21), respectively. $T=300 \mathrm{~K}, N_{e}=10^{12} \mathrm{~cm}^{-2}, N_{s}=$ $2.1 \times 10^{12} \mathrm{~cm}^{-2}$ and $N_{\mathrm{Au}}=0$.

\section{CONCLUSION}

In conclusion, we have investigated the spin diffusion and transport in graphene monolayer on $\mathrm{SiO}_{2}$ substrate as presented by Pi et al., ${ }^{31}$ by means of the KSBE approach. The substrate (including the impurities initially present) contributes a Rashba spin-orbit coupling field much stronger than the one modulated by the electric field perpendicular to the graphene layer. By surface chemical doping with $\mathrm{Au}$ adatoms, the Rashba spin-orbit coupling coefficient $\alpha_{\mathrm{R}}$ is increased. By fitting the chemical doping dependence of diffusion coefficient and spin relaxation time, ${ }^{31}$ we obtain the information on impurities as well as the chemical doping dependence of $\alpha_{\mathrm{R}}$. Our fitting finds that $\alpha_{\mathrm{R}}$ increases linearly from 0.15 to $0.23 \mathrm{meV}$ with increasing $\mathrm{Au}$ density when the latter is not so high. With the necessary parameters obtained from fitting, we investigate the spin diffusion and transport in graphene both analytically and numerically.

The analytical study with only the electron-impurity scattering included reveals that in the strong scattering limit (just as the situation under investigation in the present work), the spin diffusion is uniquely determined by $\alpha_{R}$. When the injected spins are polarized along the $x$-, $y$ - and $z$-axis, the spin diffusion lengths are given by the analytical study with an anisotropy as $l_{x}=l_{z} \approx 0.74 \hbar v_{\mathrm{F}} / \alpha_{\mathrm{R}}$ and $l_{y}=0.5 \hbar v_{\mathrm{F}} / \alpha_{\mathrm{R}}$. Meanwhile, the spatial spin precession is present when the injected spins are polarized in the $x-z$ plane but absent when the injected spins are polarized along the $y$-axis. Further 


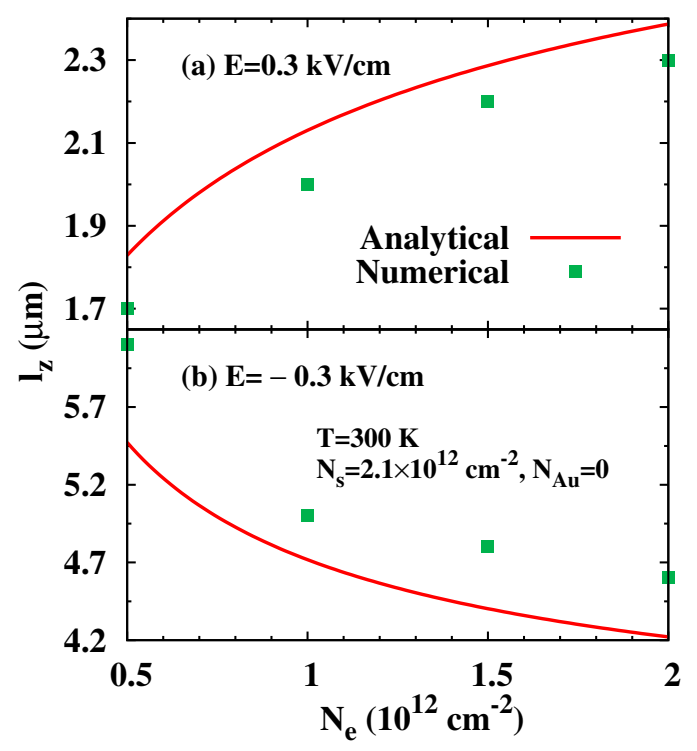

FIG. 7: (Color online) Electron density dependence of spin transport length $l_{z}$ under electric fields with opposite directions: (a) $E=0.3 \mathrm{kV} / \mathrm{cm}$ and (b) $E=-0.3 \mathrm{kV} / \mathrm{cm}$. The squares are from the numerical calculation while the curves are from Eq. (21). $T=300 \mathrm{~K}, N_{s}=2.1 \times 10^{12} \mathrm{~cm}^{-2}$ and $N_{\mathrm{Au}}=0$.

numerical calculations with all the scattering explicitly included show that the analytical model depicts the spin diffusion pretty well.

It is noted that the anisotropy of spin diffusion length from the KSBEs differs from the one from the twocomponent drift-diffusion model where $l_{x}=l_{y}=\sqrt{2} l_{z}=$ $0.5 \hbar v_{\mathrm{F}} / \alpha_{\mathrm{R}}$. The qualitative discrepancy indicates the inadequacy of the two-component drift-diffusion model due to the neglect of the off-diagonal spin components, i.e., the spin coherence. In fact, only when the injected spins are polarized along the $y$-axis and the spatial spin precession is absent, the two-component drift-diffusion model gives the same spin diffusion length as the KSBE approach does.

The analytical and numerical study of spin transport under an electric field parallel or antiparallel to the spin injection direction is also investigated. In the presence of the electric field, the analytical model depicts the spin transport with a small discrepancy which increases with the strength of the electric field. It is shown that when the electric field is applied, the spin precession in spatial domain for the cases with the injected spins polarized along the $x$ - and $z$-axis remains almost unchanged. However, the spin transport length is increased (decreased) by increasing the magnitude of the electric field when it is antiparallel (parallel) to the spin transport direction. Moreover, in the presence of the electric field, the spin transport becomes sensitive to the electron density, differing from the case of spin diffusion. The spin transport is enhanced (suppressed) by increasing electron density when the electric field is parallel (antiparallel) to the spin injection direction.

\section{Acknowledgments}

This work was supported by the National Natural Science Foundation of China under Grant No. 10725417. One of the authors (MWW) acknowledges valuable discussions with J. Fabian and B. J. van Wees.

\section{Appendix A: Spin relaxation in graphene}

We consider spin relaxation in graphene under the spatial uniform case in the absence of the electric field. We only include the electron-impurity scattering. The KSBEs, Eq. (5), are then simplified to be

$$
\begin{aligned}
\partial_{t} \rho_{\mu \mathbf{k}}(t)= & -\frac{i}{\hbar}\left[\boldsymbol{\Omega}_{\mathbf{k}} \cdot \boldsymbol{\sigma}, \rho_{\mu \mathbf{k}}(t)\right]-\frac{2 \pi}{\hbar} \sum_{\mathbf{k}^{\prime}} M_{\mathbf{k}-\mathbf{k}^{\prime}} I_{\mathbf{k} \mathbf{k}^{\prime}} \\
& \times \delta\left(\varepsilon_{\mathbf{k}}-\varepsilon_{\mathbf{k}^{\prime}}\right)\left[\rho_{\mu \mathbf{k}}(t)-\rho_{\mu \mathbf{k}^{\prime}}(t)\right] . \quad(\mathrm{A} 1)
\end{aligned}
$$

Here $M_{\mathbf{k}-\mathbf{k}^{\prime}}=\left|U_{\mathbf{k}-\mathbf{k}^{\prime}}^{s}\right|^{2}+\left|U_{\mathbf{k}-\mathbf{k}^{\prime}}^{\mathrm{Au}}\right|^{2}$ is the total electronimpurity scattering matrix element contributed by impurities in the substrate and Au adatoms. $I_{\mathbf{k k}^{\prime}}=\frac{1}{2}[1+$ $\left.\cos \left(\theta_{\mathbf{k}}-\theta_{\mathbf{k}^{\prime}}\right)\right]$ is the form factor. ${ }^{25}$ By expanding $\rho_{\mu \mathbf{k}}(t)$ as $\rho_{\mu \mathbf{k}}(t)=\sum_{l} \rho_{\mu k}^{l}(t) e^{i l \theta_{\mathbf{k}}}$, one comes to

$$
\begin{aligned}
\partial_{t} \rho_{\mu k}^{l}(t)= & -\frac{\alpha_{\mathrm{R}}}{2 \hbar}\left[\sigma_{+}, \rho_{\mu k}^{l+1}(t)\right]+\frac{\alpha_{\mathrm{R}}}{2 \hbar}\left[\sigma_{-}, \rho_{\mu k}^{l-1}(t)\right] \\
& -\frac{\rho_{\mu k}^{l}(t)}{\tau_{k}^{l}},
\end{aligned}
$$

where $\sigma_{ \pm}=\sigma_{x} \pm i \sigma_{y}$, and

$$
\frac{1}{\tau_{k}^{l}}=\frac{k\left(1-\delta_{l 0}\right)}{4 \pi \hbar^{2} v_{\mathrm{F}}} \int_{0}^{2 \pi} d \theta M_{\mathbf{q}}(1+\cos \theta)(1-\cos l \theta)
$$

with $M_{\mathbf{q}}$ depending only on $|\mathbf{q}|=2 k \sin \frac{\theta}{2}$. It is noted that $\frac{1}{\tau_{k}^{l}}=\frac{1}{\tau_{k}^{-l}}$.

Retaining the lowest three orders of $\rho_{\mu k}^{l}(t)$, i.e., $l=0$, \pm 1 , and using the initial conditions $\rho_{\mu k}^{l}(0)=\delta_{l 0} \rho_{\mu k}^{0}(0)$, one obtains the second-order differential equation about $\rho_{\mu k}^{0}(t)$ as

$$
\begin{aligned}
& \partial_{t}^{2} \rho_{\mu k}^{0}(t)+\frac{1}{\tau_{k}^{1}} \partial_{t} \rho_{\mu k}^{0}(t)+\frac{\alpha_{\mathrm{R}}^{2}}{2 \hbar^{2}}\left[\sigma_{x},\left[\sigma_{x}, \rho_{\mu k}^{0}(t)\right]\right] \\
& +\frac{\alpha_{\mathrm{R}}^{2}}{2 \hbar^{2}}\left[\sigma_{y},\left[\sigma_{y}, \rho_{\mu k}^{0}(t)\right]\right]=0
\end{aligned}
$$

with an affiliated initial condition $\partial_{t} \rho_{\mu k}^{0}(0)=0$. Defining the spin vector as $\mathbf{S}_{\mu k}^{0}(t)=\operatorname{Tr}\left[\rho_{\mu k}^{0}(t) \boldsymbol{\sigma}\right]$, one can obtain an equation satisfied by $\mathbf{S}_{\mu k}^{0}(t)$ directly from the above one, which reads

$$
\left[\partial_{t}^{2}+\frac{1}{\tau_{k}^{1}} \partial_{t}+\frac{2 \alpha_{\mathrm{R}}^{2}}{\hbar^{2}}\left(1+\delta_{\alpha z}\right)\right] S_{\mu k \alpha}^{0}(t)=0
$$


with $\alpha=x, y, z$. With the initial condition $\partial_{t} S_{\mu k \alpha}^{0}(0)=$ $0, S_{\mu k \alpha}^{0}(t)$ is solved to be

$$
\begin{aligned}
S_{\mu k \alpha}^{0}(t)= & \frac{S_{\mu k \alpha}^{0}(0)}{2}\left[\left(1+\frac{1}{\sqrt{1-c_{\alpha}^{2}}}\right) e^{-\frac{t}{2 \tau_{k}^{1}}\left(1-\sqrt{1-c_{\alpha}^{2}}\right)}\right. \\
& \left.+\left(1-\frac{1}{\sqrt{1-c_{\alpha}^{2}}}\right) e^{-\frac{t}{2 \tau_{k}^{1}}\left(1+\sqrt{1-c_{\alpha}^{2}}\right)}\right], \quad(\mathrm{A} 6)
\end{aligned}
$$

where $c_{\alpha}=2 \sqrt{2\left(1+\delta_{\alpha z}\right)} \alpha_{\mathrm{R}} \tau_{k}^{1} / \hbar$. When the scattering is strong enough and hence $c_{\alpha} \ll 1$,

$$
\begin{aligned}
S_{\mu k \alpha}^{0}(t) & \approx S_{\mu k \alpha}^{0}(0) e^{-\frac{t}{4 \tau_{k}^{1} / c_{\alpha}^{2}}} \\
& \equiv S_{\mu k \alpha}^{0}(0) e^{-\frac{t}{\tau_{\alpha}}} .
\end{aligned}
$$

As a result, for spins polarized along the $x$ - and $y$-axis the spin relaxation times are $\tau_{x}=\tau_{y}=$ $\hbar^{2} /\left(2 \alpha_{\mathrm{R}}^{2} \tau_{k}^{1}\right)$, while for spins polarized along the $z$-axis $\tau_{z}=\hbar^{2} /\left(4 \alpha_{\mathrm{R}}^{2} \tau_{k}^{1}\right)$. From Eq. (A3) one notices that $\tau_{k}^{1}$ is in fact the momentum relaxation time $\tau_{p}(k)$. For the highly degenerate electron system in graphene, $\tau_{p}(k) \approx$ $\tau_{p}\left(k_{\mathrm{F}}\right) \approx \tau_{p}$. Therefore we have $\tau_{x}=\tau_{y}=2 \tau_{z}=$ $\hbar^{2} /\left(2 \alpha_{\mathrm{R}}^{2} \tau_{p}\right)$.

\section{Appendix B: Spin diffusion in graphene}

The spin diffusion in the absence of an electric field is also investigated for the case with only the electronimpurity scattering included. Performing angle expansion on the steady-state KSBEs in a way similar to that shown in Appendix A, one arrives at

$$
\begin{aligned}
& \partial_{x} \sum_{l_{0}= \pm 1} \rho_{\mu k}^{l+l_{0}}(x)+\gamma\left[\sigma_{+}, \rho_{\mu k}^{l+1}(x)\right]-\gamma\left[\sigma_{-}, \rho_{\mu k}^{l-1}(x)\right] \\
& +\frac{2}{v_{\mathrm{F}}} \frac{\rho_{\mu k}^{l}(x)}{\tau_{k}^{l}}=0
\end{aligned}
$$

with $\gamma=\alpha_{\mathrm{R}} /\left(\hbar v_{\mathrm{F}}\right)$. Retaining the lowest three orders of $\rho_{\mu k}^{l}(x)$ one obtains three equations involving $\rho_{\mu k}^{0, \pm 1}(x)$ as

$$
\begin{aligned}
& \partial_{x} \sum_{l_{0}= \pm 1} \rho_{\mu k}^{l_{0}}(x)+\gamma\left[\sigma_{+}, \rho_{\mu k}^{1}(x)\right]-\gamma\left[\sigma_{-}, \rho_{\mu k}^{-1}(x)\right]=0, \\
& \partial_{x} \rho_{\mu k}^{0}(x)-\gamma\left[\sigma_{-}, \rho_{\mu k}^{0}(x)\right]+\frac{2}{v_{\mathrm{F}}} \frac{\rho_{\mu k}^{1}(x)}{\tau_{k}^{1}}=0 \\
& \partial_{x} \rho_{\mu k}^{0}(x)+\gamma\left[\sigma_{+}, \rho_{\mu k}^{0}(x)\right]+\frac{2}{v_{\mathrm{F}}} \frac{\rho_{\mu k}^{-1}(x)}{\tau_{k}^{1}}=0
\end{aligned}
$$

From these equations one immediately arrives at Eq. (7) with $\tau_{k}^{1}$ being irrelevant. By multiplying $\boldsymbol{\sigma}$ and performing trace on both sides of Eq. (7), one gets the equation satisfied by $\mathbf{S}_{\mu k}^{0}(x)$ which can be written as

$$
\left(\begin{array}{ccc}
\partial_{x}^{2}-4 \gamma^{2} & 0 & -4 \gamma \partial_{x} \\
0 & \partial_{x}^{2}-4 \gamma^{2} & 0 \\
4 \gamma \partial_{x} & 0 & \partial_{x}^{2}-8 \gamma^{2}
\end{array}\right)\left(\begin{array}{c}
S_{\mu k x}^{0}(x) \\
S_{\mu k y}^{0}(x) \\
S_{\mu k z}^{0}(x)
\end{array}\right)=0
$$

With specified boundary conditions, $\mathbf{S}_{\mu k}^{0}(x)$ is solved and the total spin signal $\mathbf{S}(x)$ is obtained by Eq. (8), as presented in Sec. III B 1. Explicitly, taking the boundary condition (I) given in Sec. III B 1 as an example, one obtains $\mathbf{S}_{\mu k}^{0}(x)$ as

$$
\mathbf{S}_{\mu \mathbf{k}}^{0}(x)=S_{\mu k}^{0}(0) e^{-x / l_{x}}\left(\begin{array}{c}
\sqrt{1+\Delta^{2}} \sin (\omega x+\phi) \\
0 \\
c_{1} \sin (\omega x)
\end{array}\right)
$$

with the parameters $l_{x}, \omega, \Delta, \phi$ and $c_{1}$ given in Sec. III B 1. By further summing over $\mathbf{k}$ and $\mu$ one arrives at Eq. (9).

\section{Appendix C: Spin transport in graphene}

The analytical study of spin transport is carried out analogly. The driving term from the electric field in the steady state is approximated as

$$
\begin{aligned}
\frac{e E}{\hbar} \frac{\partial \rho_{\mu \mathbf{k}}(x)}{\partial k_{x}} & =\frac{e E}{\hbar} \frac{\partial \rho_{\mu \mathbf{k}}(x)}{\partial \varepsilon_{\mathbf{k}}} \frac{\partial \varepsilon_{\mathbf{k}}}{\partial k_{x}} \\
& \approx e E v_{\mathrm{F}} \cos \theta_{\mathbf{k}} \frac{\partial \rho_{\mu k}^{0}(x)}{\partial \varepsilon_{\mathbf{k}}} .
\end{aligned}
$$

Then the Fourier transformation of the steady-state KSBEs reads

$$
\begin{gathered}
\partial_{x} \sum_{l_{0}= \pm 1} \rho_{\mu k}^{l+l_{0}}(x)+\gamma\left[\sigma_{+}, \rho_{\mu k}^{l+1}(x)\right]-\gamma\left[\sigma_{-}, \rho_{\mu k}^{l-1}(x)\right] \\
-e E \frac{\partial \rho_{\mu k}^{0}(x)}{\partial \varepsilon_{\mathbf{k}}}\left(\delta_{l-1}+\delta_{l 1}\right)+\frac{2}{v_{\mathrm{F}}} \frac{\rho_{\mu k}^{l}(x)}{\tau_{k}^{l}}=0 .
\end{gathered}
$$

From this equation one comes to Eq. (20) by retaining the lowest three orders of $\rho_{\mu k}^{l}(x)$. The equation satisfied by $\mathbf{S}_{\mu k}^{0}(x)$ is then

$$
\left(\begin{array}{ccc}
\partial_{x}^{2}-e E \partial_{x} \partial_{\varepsilon_{\mathbf{k}}}-4 \gamma^{2} & 0 & -4 \gamma \partial_{x}+2 e E \gamma \partial_{\varepsilon_{\mathbf{k}}} \\
0 & \partial_{x}^{2}-e E \partial_{x} \partial_{\varepsilon_{\mathbf{k}}}-4 \gamma^{2} & 0 \\
4 \gamma \partial_{x}-2 e E \gamma \partial_{\varepsilon_{\mathbf{k}}} & 0 & \partial_{x}^{2}-e E \partial_{x} \partial_{\varepsilon_{\mathbf{k}}}-8 \gamma^{2}
\end{array}\right)\left(\begin{array}{c}
S_{\mu k x}^{0}(x) \\
S_{\mu k y}^{0}(x) \\
S_{\mu k z}^{0}(x)
\end{array}\right)=0
$$


Having the experience of solving Eq. (B5), we assume that $\mathbf{S}_{\mu k}^{0}(x)$ has the solution as $\mathbf{S}_{\mu k}^{0}(x)=S_{\mu k}^{0}(0) \mathbf{T}(x)$ and therefore $\mathbf{S}(x)=S(0) \mathbf{T}(x)$. Performing summation over $\mu$ and $\mathbf{k}$ on both sides of the above equation and using the trick

$$
\begin{aligned}
\int_{0}^{+\infty} d k k\left[\partial_{\varepsilon_{\mathbf{k}}} S_{\mu k}^{0}(0)\right] \mathbf{T}(x) & =-\frac{\int_{0}^{+\infty} d \varepsilon_{\mathbf{k}} S_{\mu k}^{0}(0)}{\hbar^{2} v_{\mathrm{F}}^{2}} \mathbf{T}(x) \\
& =-\frac{\frac{1}{\beta} \ln \frac{1+e^{\beta \mu_{\uparrow}}}{1+e^{\beta \mu_{\downarrow}}}}{S(0) \hbar^{2} v_{\mathrm{F}}^{2}} \mathbf{S}(x), \quad(\mathrm{C} 4)
\end{aligned}
$$

one obtains the equation satisfied by $\mathbf{S}(x)$ as

$$
\begin{aligned}
& \left(\begin{array}{ccc}
\partial_{x}^{2}+\gamma \mathscr{E} \partial_{x}-4 \gamma^{2} & 0 & -4 \gamma \partial_{x}-2 \gamma^{2} \mathscr{E} \\
0 & \partial_{x}^{2}+\gamma \mathscr{E} \partial_{x}-4 \gamma^{2} & 0 \\
4 \gamma \partial_{x}+2 \gamma^{2} \mathscr{E} & 0 & \partial_{x}^{2}+\gamma \mathscr{E} \partial_{x}-8 \gamma^{2}
\end{array}\right) \\
& \times\left(\begin{array}{c}
S_{x}(x) \\
S_{y}(x) \\
S_{z}(x)
\end{array}\right)=0,
\end{aligned}
$$

in which $\mathscr{E}$ is given by Eq. (24). With the three different typical boundary conditions presented in Sec. III B 1, $\mathbf{S}(x)$ is solved to have the same form as Eqs. (9)-(11) except that the parameters are now given in Sec. III B 2.

We now calculate $\mathscr{E}$ in both the degenerate and nondegenerate limits. In the degenerate limit, $\ln \frac{1+e^{\beta \mu_{\uparrow}}}{1+e^{\beta \mu_{\downarrow}}} \approx \ln e^{\beta\left(\varepsilon_{\mathrm{F} \uparrow}-\varepsilon_{\mathrm{F} \downarrow}\right)}=\beta \hbar v_{\mathrm{F}}\left(k_{\mathrm{F} \uparrow}-k_{\mathrm{F} \downarrow}\right)=$ $\beta \hbar v_{\mathrm{F}} \sqrt{\pi N_{e}}\left(\sqrt{1+P_{0}}-\sqrt{1-P_{0}}\right) \approx \beta \hbar v_{\mathrm{F}} \sqrt{\pi N_{e}} P_{0}$. Making use of the relation $S(0)=N_{e} P_{0}$, one has

$$
\mathscr{E} \approx \frac{e E}{\sqrt{\pi N_{e}} \alpha_{\mathrm{R}}}=\frac{e E}{\alpha_{\mathrm{R}} k_{\mathrm{F}}},
$$

where $k_{\mathrm{F}}=\sqrt{\pi N_{e}}$, the magnitude of Fermi momentum of unpolarized electrons with density being $N_{e}$. In the nondegenerate limit, $\ln \frac{1+e^{\beta \mu_{\uparrow}}}{1+e^{\beta \mu_{\downarrow}}} \approx e^{\beta \mu_{\uparrow}}-e^{\beta \mu_{\downarrow}}$ and

$$
\begin{aligned}
S(0) & \approx \frac{1}{\pi} \int_{0}^{+\infty} d k k\left[e^{-\beta\left(\varepsilon_{\mathbf{k}}-\mu_{\uparrow}\right)}-e^{-\beta\left(\varepsilon_{\mathbf{k}}-\mu_{\downarrow}\right)}\right] \\
& =\frac{1}{\pi\left(\beta \hbar v_{\mathrm{F}}\right)^{2}}\left(e^{\beta \mu_{\uparrow}}-e^{\beta \mu_{\downarrow}}\right),
\end{aligned}
$$

therefore

$$
\mathscr{E} \approx \frac{e E \beta \hbar v_{\mathrm{F}}}{\alpha_{\mathrm{R}}} .
$$

\section{Appendix D: Derivation of two-component drift-diffusion equation from KSBEs}

The two-component drift-diffusion equation can be derived from the KSBEs in the collinear spin space ${ }^{57}$ with the $z$-axis along the initial spin-polarization direction $\hat{\mathbf{n}}$, by neglecting the spin coherence (i.e., the off-diagonal components of the density matrices). The density matrices then have the diagonal form as $\frac{1}{2}\left[f_{\mu \mathbf{k} \uparrow}(x, t)+\right.$ $\left.f_{\mu \mathbf{k} \downarrow}(x, t)+\left(f_{\mu \mathbf{k} \uparrow}(x, t)-f_{\mu \mathbf{k} \downarrow}(x, t)\right) \sigma_{z}\right]$. In the following we present a brief derivation of the two-component driftdiffusion equation from the KSBEs with only the strong electron-impurity scattering considered. Other kinds of scattering can also be incorporated similarly under elastic scattering approximation. The spin relaxation time is $\tau_{\hat{\mathbf{n}}}$ and the momentum relaxation time is $\tau_{p}$ (both are given in Appendix A) and we neglect their momentum dependence hereafter. The two-component drift-diffusion equation is obtained from the equation of continuity and the equation of current, both to be derived from the KSBEs.

The equation of continuity is derived as follows. By multiplying the KSBEs [Eq. (5)] with $\frac{1}{2}\left(1 \pm \sigma_{z}\right)$ and then performing the trace, one obtains the simplified KSBEs for each spin band $(\sigma=\uparrow, \downarrow)$ as

$$
\begin{aligned}
& \frac{\partial f_{\mu \mathbf{k} \sigma}(x, t)}{\partial t}-\frac{e E}{\hbar} \frac{\partial f_{\mu \mathbf{k} \sigma}(x, t)}{\partial k_{x}}+v_{\mathrm{F}} \cos \theta_{\mathbf{k}} \frac{\partial f_{\mu \mathbf{k} \sigma}(x, t)}{\partial x} \\
& =-\frac{f_{\mu \mathbf{k} \sigma}(x, t)-f_{\mu \mathbf{k}-\sigma}(x, t)}{2 \tau_{\hat{\mathbf{n}}}} .
\end{aligned}
$$

The right-hand side of the above equation comes from the term $\operatorname{Tr}\left\{\frac{1}{2}\left(1 \pm \sigma_{z}\right)\left[\left.\partial_{t} \rho_{\mu \mathbf{k}}(x, t)\right|_{\text {coh }}+\left.\partial_{t} \rho_{\mu \mathbf{k}}(x, t)\right|_{\text {scat }}\right]\right\}$, which can be calculated with the aid of the KSBEs in the time domain [Eq. (A1)] and the corresponding solution [Eq. (A7)]. Performing summation over $\mu$ and $\mathbf{k}$ on both sides of Eq. (D1) in the steady state, one comes to

$$
\begin{aligned}
& -\frac{e E}{\hbar} \sum_{\mu \mathbf{k}} \frac{\partial f_{\mu \mathbf{k} \sigma}(x)}{\partial k_{x}}+\frac{\partial}{\partial x} \sum_{\mu \mathbf{k}} v_{\mathrm{F}} \cos \theta_{\mathbf{k}} f_{\mu \mathbf{k} \sigma}(x) \\
& =-\frac{N_{\sigma}(x)-N_{-\sigma}(x)}{2 \tau_{\hat{\mathbf{n}}}}
\end{aligned}
$$

where $N_{\sigma}(x)$ is the electron density with spin $\sigma$ at position $x$. Up to the first order of the electric field $E$, the first summation over $\mathbf{k}$ in the above equation leads to zero when $f_{\mu \mathbf{k} \sigma}$ is approximated by $f_{\mu \mathbf{k} \sigma}^{0}$, the distribution in equilibrium. Defining the charge current along the $x$-axis with spin $\sigma$ as

$$
J_{\sigma}(x)=-\sum_{\mu \mathbf{k}} e v_{x} f_{\mu \mathbf{k} \sigma}(x)
$$

with $v_{x}=v_{\mathrm{F}} \cos \theta_{\mathbf{k}}$, one has the equation of continuity

$$
-\frac{1}{e} \frac{\partial J_{\sigma}(x)}{\partial x}=-\frac{N_{\sigma}(x)-N_{-\sigma}(x)}{2 \tau_{\hat{\mathbf{n}}}} .
$$

We then calculate the current $J_{\sigma}$ from the diagonal part of the KSBEs

$$
\begin{aligned}
& \frac{\partial f_{\mu \mathbf{k} \sigma}(x, t)}{\partial t}-\frac{e E}{\hbar} \frac{\partial f_{\mu \mathbf{k} \sigma}(x, t)}{\partial k_{x}}+v_{\mathrm{F}} \cos \theta_{\mathbf{k}} \frac{\partial f_{\mu \mathbf{k} \sigma}(x, t)}{\partial x} \\
& =-\frac{f_{\mu \mathbf{k} \sigma}(x, t)-f_{\mu \mathbf{k} \sigma}^{0}(x, t)}{\tau_{p}},
\end{aligned}
$$


where the right-hand side of the equation comes from the electron-impurity scattering (Appendix A). In the steady state, multiplying $-e v_{x}$ on both sides of the equation and then summing over $\mu$ and $\mathbf{k}$, one comes to

$$
\begin{aligned}
& \frac{e^{2} E v_{\mathrm{F}}}{\hbar} \sum_{\mu \mathbf{k}} \cos \theta_{\mathbf{k}} \frac{\partial f_{\mu \mathbf{k} \sigma}(x)}{\partial k_{x}}-e v_{\mathrm{F}}^{2} \sum_{\mu \mathbf{k}} \cos ^{2} \theta_{\mathbf{k}} \frac{\partial f_{\mu \mathbf{k} \sigma}(x)}{\partial x} \\
& =-\frac{J_{\sigma}(x)}{\tau_{p}} .
\end{aligned}
$$

Again, up to the first order of the electric field, one has

$$
J_{\sigma}(x)=e \mu_{\sigma} E N_{\sigma}(x)+e D \partial_{x} N_{\sigma}(x)
$$

where the mobility $\mu_{\sigma}=\frac{e v_{\mathrm{F}} \tau_{p}}{\hbar \sqrt{2 \pi N_{\sigma}}}$ and the charge diffusion coefficient $D=\frac{1}{2} v_{\mathrm{F}}^{2} \tau_{p}$. For the case with small spin polarization, $\mu_{\uparrow} \approx \mu_{\downarrow}=\mu_{e} \equiv \frac{e v_{\mathrm{F}} \tau_{p}}{\hbar \sqrt{\pi N_{e}}}$ in which $N_{e}=N_{\uparrow}+N_{\downarrow}$ is the total electron density.

Finally, the two-component drift-diffusion equation is obtained by combining Eqs. (D4) and (D7)

$$
-\mu_{e} E \frac{\partial N_{\sigma}(x)}{\partial x}-D \frac{\partial^{2} N_{\sigma}(x)}{\partial x^{2}}=-\frac{N_{\sigma}(x)-N_{-\sigma}(x)}{2 \tau_{\hat{\mathbf{n}}}} .
$$

This equation is consistent with that in the literature. $^{44-47}$ The equation of $\Delta N=N_{\uparrow}-N_{\downarrow}$ then reads

$$
-\mu_{e} E \frac{\partial \Delta N(x)}{\partial x}-D \frac{\partial^{2} \Delta N(x)}{\partial x^{2}}=-\frac{\Delta N(x)}{\tau_{\hat{\mathbf{n}}}} .
$$

With boundary condition $\Delta N(+\infty)=0, \Delta N(x)$ is solved as $\Delta N(x)=\Delta N(0) e^{-x / l_{\hat{\mathbf{n}}}}$ where the spin transport length ${ }^{44}$

$$
l_{\hat{\mathbf{n}}}=\left[\frac{\mu_{e} E}{2 D}+\sqrt{\left(\frac{\mu_{e} E}{2 D}\right)^{2}+\frac{1}{D \tau_{\hat{\mathbf{n}}}}}\right]^{-1} .
$$

When the electric field is zero,

$$
l_{\hat{\mathbf{n}}}=\sqrt{D \tau_{\hat{\mathbf{n}}}}
$$

As indicated by Eqs. (D10) and (D11), in the frame of the two-component drift-diffusion model, the anisotropy of the spin transport or diffusion is solely determined by that of the spin relaxation and no spatial spin precession can be obtained.
* Author to whom correspondence should be addressed; Electronic address: mwwu@ustc.edu.cn.

1 A. K. Geim and K. S. Novelia, Nature Mater. 6, 183 (2007).

2 N. Tombros, C. Józsa, M. Popinciuc, H. T. Jonkman, and B. J. van Wees, Nature (London) 448, 571 (2007).

3 A. H. C. Neto, F. Guinea, N. M. R. Peres, K. S. Novoselov, and A. K. Geim, Rev. Mod. Phys. 81, 109 (2009).

4 E. H. Hang, S. Adam, and S. Das Sarma, Phys. Rev. Lett. 98, 186806 (2007).

${ }^{5}$ K. S. Novoselov, A. K. Geim, S. V. Morozov, D. Jiang, M. I. Katsnelson, I. V. Grigorieva, S. V. Dubonos and A. A. Firsov, Nature 438, 197 (2005).

${ }^{6}$ K. S. Novoselov, A. K. Geim, S. V. Morozov, D. Jiang, Y. Zhang, S. V. Dubonos, I. V. Grigorieva, and A. A. Firsov, Science 306, 666 (2004).

7 K. I. Bolotin, K.J. Sikes, Z. Jiang, M. Klima, G. Fudenberg, J. Hone, P. Kim and H. L. Stormer, Sol. State Commun. 146, 351 (2008).

8 J. Fischer, B. Trauzettel, and D. Loss, Phys. Rev. B 80, 155401 (2009).

9 C. L. Kane and E. J. Mele, Phys. Rev. Lett. 95, 226801 (2005).

10 D. H. Hernando, F. Guinea, and A. Brataas, Phys. Rev. B 74, 155426 (2006).

11 H. Min, J. E. Hill, N. A. Sinitsyn, B. R. Sahu, L. Kleinman, and A. H. MacDonald, Phys. Rev. B 74, 165310 (2006).

12 M. Gmitra, S. Konschuh, C. Ertler, C. A. Draxl, and J. Fabian, Phys. Rev. B 80, 235431 (2009).

13 C. Józsa, M. Popinciuc, N. Tombros, H. T. Jonkman, and B. J. van Wees, Phys. Rev. Lett. 100, 236603 (2008).

${ }^{14}$ H. Goto, A. Kanda, T. Sato, S. Tanaka, Y. Ootuka, S. Odaka, H. Miyazaki, K. Tsukagoshi, and Y. Aoyagi, Appl.
Phys. Lett. 92, 212110 (2008).

15 E. W. Hill, A. K. Geim, K. Novoselov, F. Schedin, and P. Blake, IEEE Trans. Mag. 42, 2694 (2006).

16 S. Cho, Y. F. Chen, and M. S. Fuhrer, Appl. Phys. Lett. 91, 123105 (2007).

17 H. Haugen, D. H. Hernando, and A. Brataas, Phys. Rev. B 77, 115406 (2008)

18 W. H. Wang, K. Pi, Y. Li, Y. F. Chiang, P. Wei, J. Shi, and R. K. Kawakami, Phys. Rev. B 77, 020402(R) (2008).

19 M. Ohishi, M. Shiraishi, R. Nouchi, T. Nozaki, T. Shinjo, and Y. Suzuki, Jpn. J. Appl. Phys. 46, L605 (2007).

${ }^{20}$ L. Brey and H. A. Fertig, Phys. Rev. B 73, 195408 (2006).

21 V. P. Gusynin and S. G. Sharapov, Phys. Rev. Lett. 95, 146801 (2005).

22 C. L. Kane and E. J. Mele, Phys. Rev. Lett. 95, 226801 (2005).

23 S. V. Morozov, K. S. Novoselov, M. I. Katsnelson, F. Schedin, D. C. Elias, J. A. Jaszczak, and A. K. Geim, Phys. Rev. Lett. 100, 016602 (2008).

24 X. Du, I. Skachko, A. Barker, and E. Y. Andrei, Nat. Nanotechnol. 3, 491 (2008).

25 Y. Zhou and M. W. Wu, Phys. Rev. B 82, 085304 (2010).

26 S. Abdelouahed, A. Ernst, and J. Henk, Phys. Rev. B 82, 125424 (2010).

27 W. Han, K. Pi, W. Bao, K. M. McCreary, Y. Li, W. H. Wang, C. N. Lau, and R. K. Kawakami, Appl. Phys. Lett. 94, 222109 (2009).

28 M. Popinciuc, C. Józsa, P. J. Zomer, N. Tombros, A. Veligura, H. T. Jonkman, and B. J. van Wees, Phys. Rev. B 80, 214427 (2009).

29 N. Tombros, S. Tanabe, A. Veligura, C. Józsa, M. Popinciuc, H. T. Jonkman, and B. J. van Wees, Phys. Rev. Lett. 
101, 046601 (2008).

30 C. Józsa, T. Maassen, M. Popinciuc, P. J. Zomer, A. Veligura, H. T. Jonkman, and B. J. van Wees, Phys. Rev. B 80, 241403(R) (2009).

31 K. Pi, W. Han, K. M. McCreary, A. G. Swartz, Y. Li, and R. K. Kawakami, Phys. Rev. Lett. 104, 187201 (2010).

32 C. Ertler, S. Konschuh, M. Gmitra, and J. Fabian, Phys. Rev. B 80, 041405(R) (2009).

33 R. J. Elliott, Phys. Rev. 96, 266 (1954).

34 A. H. C. Neto and F. Guinea, Phys. Rev. Lett. 103, 026804 (2009).

35 A. Varykhalov, J. S. Barriga, A. M. Shikin, C. Biswas, E. Vescovo, A. Rybkin, D. Marchenko, and O. Rader, Phys. Rev. Lett. 101, 157601 (2008).

${ }^{36}$ M. I. D'yakonov and V. I. Perel', Zh. Éksp. Teor. Fiz. 60, 1954 (1971) [Sov. Phys. JETP 33, 1053 (1971)].

37 M. W. Wu, J. H. Jiang, and M. Q. Weng, Phys. Rep. 493, $61(2010)$.

38 M. Q. Weng and M. W. Wu, Phys. Rev. B 66, 235109 (2002).

39 J. L. Cheng and M. W. Wu, J. Appl. Phys. 101, 073702 (2007).

40 J. L. Cheng, M. W. Wu, and I. C. da Cunha Lima, Phys. Rev. B 75, 205328 (2007).

41 P. Zhang and M. W. Wu, Phys. Rev. B 79, 075303 (2009).

42 B. Y. Sun, P. Zhang, and M. W. Wu, Semicond. Sci. Technol. 26, 075005 (2011).

43 Spin Electronics, edited by M. Ziese and M. J. Thornton (Springer, Berlin, 2001).
44 Z. G. Yu and M. E. Flatté, Phys. Rev. B 66, 201202(R) (2002).

45 I. Žutić, J. Fabian, and S. Das Sarma, Phys. Rev. Lett. 88, 066603 (2002).

46 J. Fabian, I. Zutić, S. Das Sarma, Phys. Rev. B 66, 165301 (2002).

47 J. Fabian, A. M. Abiague, C. Ertler, P. Stano, I. Žutić, Acta Phys. Slov. 57, 565 (2007).

48 S. Adam and S. Das Sarma, Solid State Commun. 146, 356 (2008).

49 M. R. Ramezanali, M. M. Vazifeh, R. Asgari, M. Polini, and A. H. MacDonald, J. Phys. A: Math. Theor. 42, 214015 (2009).

50 E. H. Hwang and S. Das Sarma, Phys. Rev. B 77, 115449 (2008)

51 M. Lazzeri, S. Piscanec, F. Mauri, A. C. Ferrari, and J. Robertson, Phys. Rev. Lett. 95, 236802 (2005).

52 S. Fratini and F. Guinea, Phys. Rev. B 77, 195415 (2008).

${ }^{53}$ H. Haug and A. P. Jauho, Quantum kinetics in Transport and Optics of Semiconductors (Springer, Berlin, 1998).

${ }^{54}$ Y. W. Tan, Y. Zhang, K. Bolotin, Y. Zhao, S. Adam, E. H. Hwang, S. Das Sarma, H. L. Stormer, and P. Kim, Phys. Rev. Lett. 99, 246803 (2007).

55 I. Appelbaum, B. Huang, and D. J. Monsma, Nature (London) 447, 295 (2007).

56 G. Dresselhaus, Phys. Rev. 100, 580 (1955).

57 J. L. Cheng and M. W. Wu, J. Appl. Phys. 99, 083704 (2006). 